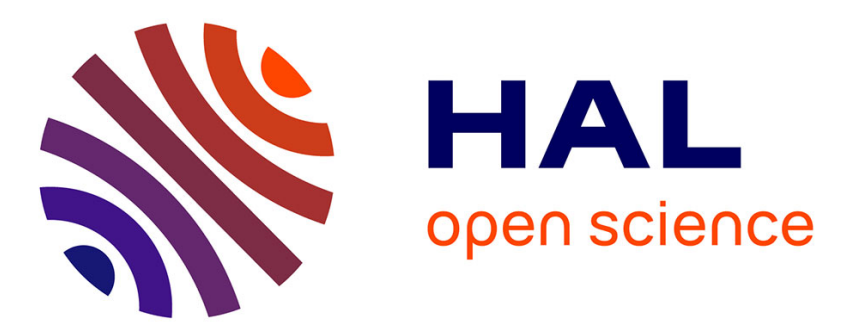

\title{
Structural Graph-Based Morphometry: a multiscale searchlight framework based on sulcal pits
}

Sylvain Takerkart, Guillaume Auzias, Lucile Brun, Olivier Coulon

\section{To cite this version:}

Sylvain Takerkart, Guillaume Auzias, Lucile Brun, Olivier Coulon. Structural Graph-Based Morphometry: a multiscale searchlight framework based on sulcal pits. Medical Image Analysis, 2017, 35, pp.32-45. 10.1016/j.media.2016.04.011 . hal-01264416v2

\section{HAL Id: hal-01264416 \\ https://hal.science/hal-01264416v2}

Submitted on 2 Mar 2017

HAL is a multi-disciplinary open access archive for the deposit and dissemination of scientific research documents, whether they are published or not. The documents may come from teaching and research institutions in France or abroad, or from public or private research centers.
L'archive ouverte pluridisciplinaire HAL, est destinée au dépôt et à la diffusion de documents scientifiques de niveau recherche, publiés ou non, émanant des établissements d'enseignement et de recherche français ou étrangers, des laboratoires publics ou privés. 


\title{
Structural Graph-Based Morphometry: a multiscale searchlight framework based on sulcal pits
}

\author{
Sylvain Takerkart ${ }^{\star a, b}$, Guillaume Auzias ${ }^{\mathrm{a}, \mathrm{c}}$, Lucile Brun ${ }^{\mathrm{a}, \mathrm{c}}$, Olivier Coulon ${ }^{\mathrm{a}, \mathrm{c}}$ \\ * Corresponding author: Sylvain.Takerkart@univ-amu.fr \\ ${ }^{a}$ Institut de Neurosciences de la Timone UMR 7289, Aix-Marseille Université, CNRS \\ Faculté de Médecine, 27 boulevard Jean Moulin, 13005 Marseille, France \\ ${ }^{b}$ Aix-Marseille Université, CNRS, Laboratoire d'Informatique Fondamentale UMR 7279 \\ Faculté des Sciences, 163 avenue de Luminy, Case 901, 13009 Marseille, France \\ ${ }^{c}$ Aix-Marseille Université, CNRS, LSIS laboratory, UMR 7296 \\ Bâtiment Polytech Saint Jérôme, Avenue Escadrille Normandie-Niemen, 13013 Marseille, France
}

\begin{abstract}
Studying the topography of the cortex has proved valuable in order to characterize populations of subjects. In particular, the recent interest towards the deepest parts of the cortical sulci - the so-called sulcal pits - has opened new avenues in that regard. In this paper, we introduce the first fully automatic brain morphometry method based on the study of the spatial organization of sulcal pits - Structural Graph-Based Morphometry (SGBM). Our framework uses attributed graphs to model local patterns of sulcal pits, and further relies on three original contributions. First, a graph kernel is defined to provide a new similarity measure between pit-graphs, with few parameters that can be efficiently estimated from the data. Secondly, we present the first searchlight scheme dedicated to brain morphometry, yielding dense information maps covering the full cortical surface. Finally, a multi-scale inference strategy is designed to jointly analyze the searchlight information maps obtained at different spatial scales. We demonstrate the effectiveness of our framework by studying gender differences and cortical asymmetries: we show that SGBM can both localize informative regions and estimate their preferred spatial scales, while providing results which are consistent with the literature. Thanks to the modular design of our kernel and the vast array of available kernel methods, SGBM can easily be extended to include a more detailed description of the sulcal patterns and solve different statistical problems. Therefore, we suggest that our SGBM framework should be useful for both reaching a better understanding of the normal brain and defining imaging biomarkers in clinical settings.
\end{abstract}


Keywords: morphometry, brain, sulcal pits, graph kernel, searchlight, multi-scale methods

\section{Introduction}

In the past few years, the topography of the cortical surface has raised a lot of interest, in particular to find biomarkers of pathologies (Im et al. (2012); Auzias et al. (2014)) or to detect features associated with functional specificities (Sun et al. (2012b)). In order to perform automatic morphometry based on the local organization of the cortex, studying high-level objects such as cortical sulci allows to alleviate the dependency on the one-to-one voxel/vertex correspondence which is required in traditional Voxel-Based and Surface-Based Morphometry (VBM, Ashburner and Friston (2000); SBM Van Essen et al. (2001)). This has led to the development of Object-Based Morphometry (OBM, Mangin et al. (2004)), in which each sulcus is described by a large set of attributes. OBM has been successfully used to characterize various populations of subjects, for instance by mapping gender differences Duchesnay et al. (2007) or by distinguishing patients from control subjects in pathologies such as schizophrenia Cachia et al. (2008) or autism Auzias et al. (2014).

More recently, a specific attention has been brought to the deepest part of sulci, either to elaborate theoretical models of cortical anatomy and development Régis et al. (2005), or to automatically extract robust cortical landmarks Meng et al. (2014); Auzias et al. (2015). For the latter, the seminal work of Lohmann et al. (2008) has been particularly important in defining the concept of sulcal pits, with follow-ups brought by Im et al. (2010) and Auzias et al. (2015). This provides a finer spatial representation than the one offered by sulci - because a single sulcus can contain several sulcal pits. In particular, modelling local patterns of sulcal pits as graphs (see Fig,2) has proved effective in order to establish links with genetic factors Lohmann et al. (2008); Im et al. (2011) or characterize groups of patients Im et al. (2012, 2015). These studies are all based on the statistical analysis of betweensubjects comparisons of pit-graphs defined within a pre-determined region of interest (ROI). These comparisons use a carefully designed similarity measure described in Im et al. (2011), that relies on a spectral matching method to define pit-to-pit correspondences between subjects.

The main limitations of this ROI-based method are the following. First, the effectiveness of the similarity measure of Im et al. (2011) strongly depends 
on the choice of the values of seven hyper-parameters which required a large set of specific experiments. Secondly, as with any ROI-based approach, one need to have strong a priori hypotheses to define the location and the size of the region to study. In that regard, the literature offers very little $a$ priori information. The few published studies that looked at cortical folding patterns using sulcal pits examined either brain lobes in Im et al. (2011, 2012) or single sulcal basins Im et al. (2010); Auzias et al. (2015). Since both these approaches made it possible to detect significant effects at very different scales, it seems necessary to systematically conduct such study over a large range of spatial scales.

In order to overcome these limitations, we here introduce Structural GraphBased Morphometry (SGBM), which is an extension of the work previously presented in Takerkart et al. (2015). This framework relies on three main contributions:

i) The design of a graph kernel that provides a new similarity measure between pit-graphs and allows - through the vast array of existing kernel methods Scholkopf and Smola (2001) - to perform various statistical analyses directly in graph space. This kernel has very few parameters that can be efficiently inferred from the data.

ii) The definition of a searchlight scheme - the first designed to perform brain morphometry - that yields information maps estimated from patterns of sulcal pits constructed at different spatial scales. Searchlight methods, introduced by Kriegeskorte et al. (2006), consist in fitting a multivariate statistical model (e.g a classifier) on patterns defined in a local neighborhood, and repeating this operation in a sliding window fashion to fully cover the brain. A summary statistic (for instance the accuracy of the classifier) is then assigned to the center of each neighborhood, thus yielding a spatial information map that allows the localization of the informative regions.

iii) The construction of a non parametric multi-scale inference strategy that facilitates this localization by jointly analyzing the searchlight information maps obtained at all scales and offers a high detection power. Multi-scale methods aim at studying phenomena for which the optimal scale to be used is unknown Koenderink (1984); Lindeberg (1994), as when studying the local organization of sulcal pits. They have been used in neuroimaging for various tasks, such as the description of activation patterns in PET (Coulon et al. (2000) ) and fMRI (Operto et al. (2012)) data or the segmentation of subcortical regions in anatomical MRI (Wu et al. (2015)). Etzel et al. (2013) also suggested that multi-scale strategies could be useful to desambiguate the 
a posteriori interpretation of regions detected by searchlight-based methods, which we implement here.

SGBM combines these three novelties in order to build a new fully automatic brain morphometry framework. This framework studies local patterns of sulcal pits by comparing them using our graph kernel, in order to define a classification-based searchlight scheme that overcomes the limitations of ROI-based approaches while embedding a multi-scale inference framework. This makes it possible to detect differences in local folding patterns at different scales between several groups of individuals. In the following, we first present the SGBM framework step by step, including a detailed description of our three contributions. We then demonstrate the power of our framework on two classical brain mapping problems for which complex patterns of anatomical differences have previously been reported in the literature: the mapping of asymmetries between the left and right brain hemispheres and the detection of cortical shape differences between male and female subjects.

\section{Methods}

\subsection{Extracting sulcal pits from T1 MR images}

In order to obtain the sulcal pits from an anatomical MR image, we first perform the segmentation of the cortical ribbon and the cortical reconstruction according to Dale et al. (1999) using the freesurfer image analysis suite

11. Then, we use the method describe in Auzias et al. (2015) to extract the sulcal pits from the cortical sheet of each subject and the Depth Potential Function of Boucher et al. (2009) to define a depth feature for each sulcal pit. This pit extraction method was carefully designed to obtain reproducible sulcal pits in every cortical region and not only in the deepest sulci, which is of critical importance to study patterns of pits centered around all cortical locations, as aimed at by the framework introduced in this paper. In order to compare pits between different subjects of the population $\mathcal{S}$ to be studied, we need to bring them into a common space. We use the cortical registration algorithm available in freesurfer Fischl et al. (1999) in order to align the convexity information across individuals after having inflated these meshes to a sphere. This sphere, onto which all subjects are aligned, will serve as our common space onto which we project the sulcal pits and basins

\footnotetext{
$1 \longdiv { \text { ww. freesurfer-software.org } }$
} 
of every subject, as illustrated on Fig. 1. For each subject $s \in \mathcal{S}$, we thus obtain the set of sulcal pits $\mathcal{P}^{s}=\left\{\pi_{i}^{s}\right\}_{i=1}^{i=N^{s}}$ localized on the sphere and their corresponding basins $\left\{\beta_{i}^{s}\right\}_{i=1}^{i=N^{s}}$. Note that the number of pits $N^{s}$ can vary across subjects.
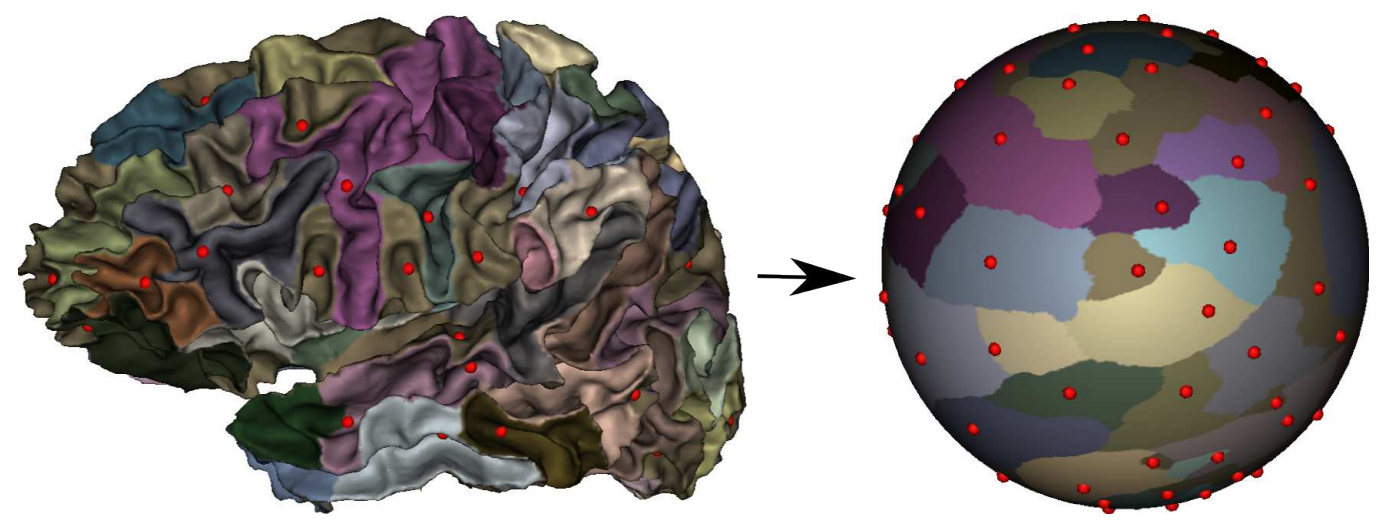

Figure 1: Left: sulcal pits of one subject, represented on the cortical sheet. Right: after cortical registration to the template and projection to the common spherical space, in which inter-subject pits locations are compared.

\subsection{Representing folding patterns as pit-graphs}

A natural way to formally represent the local folding pattern of a given part of the cortex is to construct a region adjacency graph from the set of sulcal pits and basins located within this region, as proposed in $\operatorname{Im}$ et al. (2011). Given a subset $\mathcal{P} \subset \mathcal{P}^{s}$ of $N$ pits $\left(N \leq N^{s}\right)$, each pit $\pi_{i}^{s} \in \mathcal{P}$ defines a node of the graph. The graph edges are then given by the spatial adjacency of their associated basins: this defines a binary adjacency matrix $\mathcal{A}=\left(a_{i j}\right) \in \mathbb{R}^{N \times N}$ $\left(a_{i j}=1\right.$ if $\beta_{i}$ and $\beta_{j}$ are adjacent, and 0 otherwise), that encodes the spatial organization of the folding pattern. We can then add attributes to each graph node in order to better characterize the folding pattern. In the present paper, we use two attributes for each pit: i) its euclidean coordinates on the common sphere $X_{i}$, to compare graph nodes localization across subjects, and ii) its depth $d_{i}$, because it is an intrinsic characteristic of the pit. The mapping from each subject to the common sphere explicitly minimizing metric distorsions Fischl et al. (1999), this sphere is a good space to compare pits locations across subjects in a spatially uniform manner. Note that other attributes can be added in the same manner without affecting the rest of the 
method, making these graphs a versatile representation. Let $\mathcal{D}=\left\{d_{i}\right\} \in \mathbb{R}^{N}$ be the vector of depth values and $\mathcal{X}=\left\{X_{i}\right\} \in \mathbb{R}^{N \times 3}$ be the matrix of coordinates of all graph nodes. Any pattern of sulcal pits can therefore be fully represented by an attributed graph defined as $G=(\mathcal{P}, \mathcal{A}, \mathcal{D}, \mathcal{X})$. Examples of sulcal pit graphs are shown on Fig. 2 .
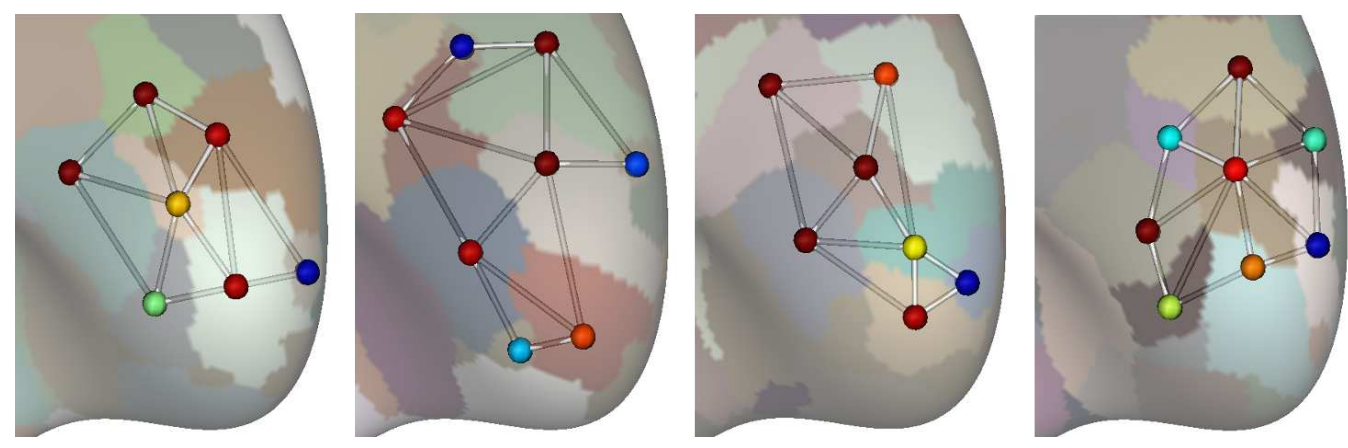

Figure 2: Sulcal pit-graphs in the right frontal lobe for four subjects, displayed on the inflated cortex. The node color encodes the depth of the pit, from blue (shallow) to red (deep).

\subsection{Measuring pattern similarity using a graph kernel}

Our first contribution is to introduce a graph kernel that provides a new similarity measure between pit-graphs. It is adapted from of a graph kernel that we designed to handle inter-subject variability in fMRI data Takerkart et al. (2014). In order to compare two pit-graphs $G=\left(\mathcal{P}^{G}, \mathcal{A}^{G}, \mathcal{D}^{G}, \mathcal{X}^{G}\right)$ and $H=\left(\mathcal{P}^{H}, \mathcal{A}^{H}, \mathcal{D}^{H}, \mathcal{X}^{H}\right)$, our kernel exploits the different characteristics of the graphs by comparing all pairs of nodes (i.e potential edges) $g_{i j}$ and $h_{k l}$, respectively in $G$ and $H$. As such, it belongs to the class of walk-based graph kernels Gärtner et al. (2003) and uses the most elementary walks, of length one. It combines (see eq. 4) the different features of the graphs by using several sub-kernels within the convolution kernel framework Haussler (1999). A first sub-kernel acts on the graph structure itself, by ensuring that the comparisons are performed only if $g_{i j}$ and $h_{k l}$ are actual edges. This is done with the linear kernel on the binary entries of the adjacency matrices:

$$
k_{a}\left(g_{i j}, h_{k l}\right)=a_{i j}^{G} \cdot a_{k l}^{H},
$$


which takes the value 1 if $a_{i j}^{G}=a_{k l}^{H}=1$ and 0 otherwise. A second sub-kernel uses a product of Gaussian kernels on the coordinates of the nodes of $g_{i j}$ and $h_{k l}$ to compare the locations of edges across subjects:

$$
k_{x}\left(g_{i j}, h_{k l}\right)=e^{-\left\|X_{i}^{G}-X_{k}^{H}\right\|^{2} / 2 \sigma_{x}^{2}} \cdot e^{-\left\|X_{j}^{G}-X_{l}^{H}\right\|^{2} / 2 \sigma_{x}^{2}} .
$$

In practice, $k_{x}$ acts as a spatial filter that weights the comparisons of edges with their proximity, thus eliminating the comparisons of edges that are far away from eachother while tolerating inter-subject variability (if edges are close, but not perfectly matched across subjects). Finally, the last sub-kernel compares the depth attributes using the same principle:

$$
k_{d}\left(g_{i j}, h_{k l}\right)=e^{-\left\|d_{i}^{G}-d_{k}^{H}\right\|^{2} / 2 \sigma_{d}^{2}} \cdot e^{-\left\|d_{j}^{G}-d_{l}^{H}\right\|^{2} / 2 \sigma_{d}^{2}}
$$

Other optional attributes that could be added to further characterize the sulcal pits would be treated in the same manner.

The full kernel is defined as the combination of all sub-kernels applied on all pairs of nodes of $G$ and $H$, which gives in our case:

$$
K(G, H)=\sum_{i, j=1}^{N_{G}} \sum_{k, l=1}^{N_{H}} k_{a}\left(g_{i j}, h_{k l}\right) \cdot k_{x}\left(g_{i j}, h_{k l}\right) \cdot k_{d}\left(g_{i j}, h_{k l}\right)
$$

Note that the number of nodes $N_{G}$ and $N_{H}$ in $G$ and $H$ can be different. We then perform the following normalization

$$
\tilde{K}(G, H)=\frac{K(G, H)}{\sqrt{K(G, G) K(H, H)}},
$$

which ensures that $\forall G, \tilde{K}(G, G)=1$, i.e that the similarity between a graph and itself does not depend on the graph.

Note that this kernel has two hyper-parameters $\sigma_{x}$ and $\sigma_{d}$, which are the bandwidths of the gaussian sub-kernels that respectively act on the coordinates and the depth features. In order to choose the values of these parameters, we use an extension of a standard practice whose effectiveness has been demonstrated in Takerkart et al. (2014): we set $\sigma_{x}$ and $\sigma_{d}$ to the median euclidean distances between the coordinates and depth attributes of the nodes in all graphs available at training time. Note that in the case where the pit-graphs represent local folding patterns, as in the rest of the paper, the value of $\sigma_{x}$ will adapt to this property of the graphs and consequently, $k_{x}$ will act as a spatial filter within the neighborhood of interest. 


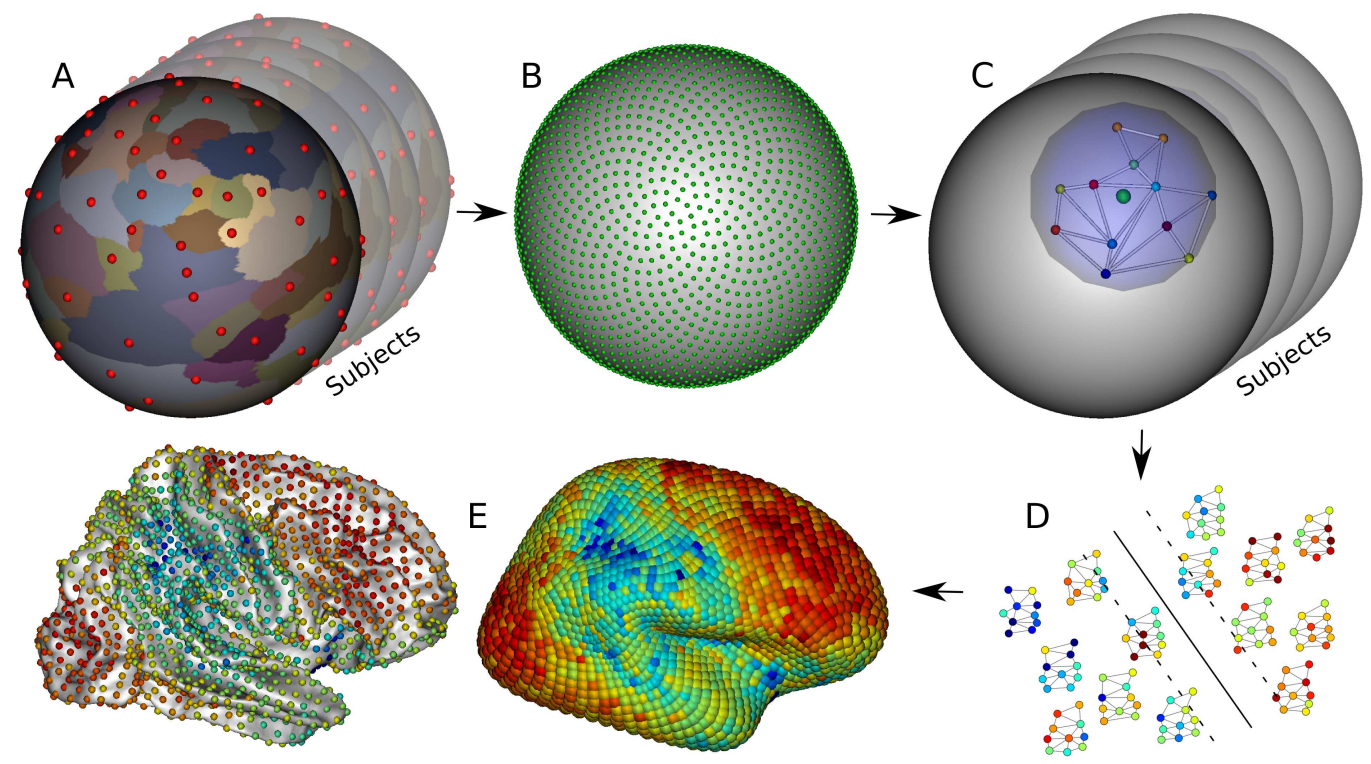

Figure 3: Illustrations of the pit-based searchlight scheme used in our SGBM framework. A: The sulcal pits and basins of a set of subjects projected onto the common spherical space. B: The Fibonacci point set used to sample the sphere. C: Around one of these points (larger green ball), the local pit-graphs defined for each subject within a spherical neiborhood (the node color encodes the depth of the sulcal pit). D: Classifying local pitgraphs into two categories (ex: patients vs. controls). E: Repeating this at all locations to obtain the searchlight information map: the Fibonacci points are projected onto the folded (left) or inflated (right) mesh (the sphere color encodes the amount of information available at this location for the classification task).

\subsection{Searchlight mapping}

Our second contribution is the definition of a searchlight method (Kriegeskorte et al. (2006)) that enables the mapping of cortical shape differences based on local folding patterns represented as sulcal pit-graphs. In order to construct a searchlight scheme for a given task, one needs to define the five following items: A: the domain of interest; B: the sampling strategy used to cover this domain; C: how to define local patterns at each location; D: the multivariate statistical model that addresses the task itself and E: the summary statistic to be used to create the information map. We now instantiate these five items, as illustrated on Fig, 3 .

In order to define our pit-based searchlight scheme in a domain where the sulcal pits of all subjects can be compared, we use the common spherical 
space where all subjects have been aligned (see 2.1) as our domain of interest (item A). Then, the Fibonacci point set (Niederreiter and Sloan (1994)) provides a pseudo-evenly distributed sampling of the sphere, with an arbitrary number of points $Q$; this defines the sampling strategy of our searchlight scheme (item B). For a given location $q \in\{1, \cdots, Q\}$ and a given subject $s \in \mathcal{S}$, we then define the set $\mathcal{P}_{q, r}^{s}$ of sulcal pits located within a radius $r$ of point $q$, i.e $\mathcal{P}_{q, r}^{s}=\left\{\pi_{i}^{s} \in \mathcal{P}^{s} \mid \operatorname{dist}\left(q, \pi_{i}^{s}\right)<r\right\}$, where dist designates the euclidean distance between two points. Given this set of pits, we can directly apply the graph construction scheme described in 2.2 to obtain the attributed graph that we note $G_{q, r}^{s}$. The set of graphs $\left\{G_{q, r}^{s}\right\}_{s \in \mathcal{S}}$ defines the local patterns at location $q$ (item $\mathrm{C}$ ) that will serve as inputs to the statistical model defined hereafter.

Equipped with our graph kernel $\tilde{K}$ defined in 2.3 , we can use any kernel method to address a wide range of problems including regression, clustering or classification. Here, we focus on supervised classification to perform group discrimination and we use a non linear Support Vector Classifier as our multivariate statistical model (item D). Such a classifier is easy to estimate by using $\tilde{K}$, which is a positive definiete kernel, and the so-called kernel trickan idea that was introduced in Aizerman et al. (1964). We then assess the generalization power of the classifier by measuring its accuracy. In practice, for a given location $q$ and a given value of $r$, we have a fully labeled dataset $\left\{\left(G_{q, r}^{s}, y_{s}\right)\right\}_{s=1}^{s=S}$ at our disposal, where the label $y_{s}$ encodes the group subject $s$ belongs to (for instance, $y_{s}=-1$ for a control subject and $y_{s}=+1$ for a patient). In order to obtain the accuracy $\bar{a}_{q}^{r}$ of the classifier, we resort to a 10-fold cross-validation which offers a good estimate of the true accuracy Kohavi (1995).

The final step in setting up a searchlight scheme consists in defining a summary statistic from the output of the statistical model in order to construct the searchlight information map. Because the distribution of $\bar{a}_{q}^{r}$ is unknown, we first resort to a permutation scheme in order to estimate it under the null hypothesis of no differences between groups, as advocated in neuroimaging in general by Bullmore et al. (1999); Nichols and Holmes (2002) and more particularly in statistical analyses of searchlight information maps by Kriegeskorte et al. (2006); Stelzer et al. (2013). Assuming the stationarity of this null distribution over the cortex, we pool together the classifier results at all searchlight locations to obtain an estimate of the cortex-wise distribution of $\bar{a}_{q}^{r}$. This provides a map of uncorrected point-wise $p$-values $\left\{p_{q}^{r}\right\}_{q \in[1, \cdots, Q]}$. Then, at each point $q$, we compute the normal statistic that 
corresponds to $p_{q}^{r}$, which we note $z_{q}^{r}$; this non linear transformation is in fact given by the so-called Inverse Error Function $e r f^{-1}$, also called Normal Inverse Cumulative Distribution function. We use this $z_{q}^{r}$-score as our summary statistic (item $\mathrm{E}$ in the definition of our searchlight scheme), which defines the searchlight information map $Z^{r}=\left\{z_{q}^{r}\right\}_{q \in[1, \cdots, Q]}$. This $Z^{r}$ map can easily be used to perform inference because we estimated its underlying distribution, and presents the nice property of being highly contrasted - as compared with the original accuracy map - thanks to the non linear transformation used to create the $z_{q}^{r}$-scores. The latter property will be useful at a later stage in our SGBM framework. The estimation of these information maps is implemented by the following algorithm, in which card designates the function that returns the size of the set given in input.

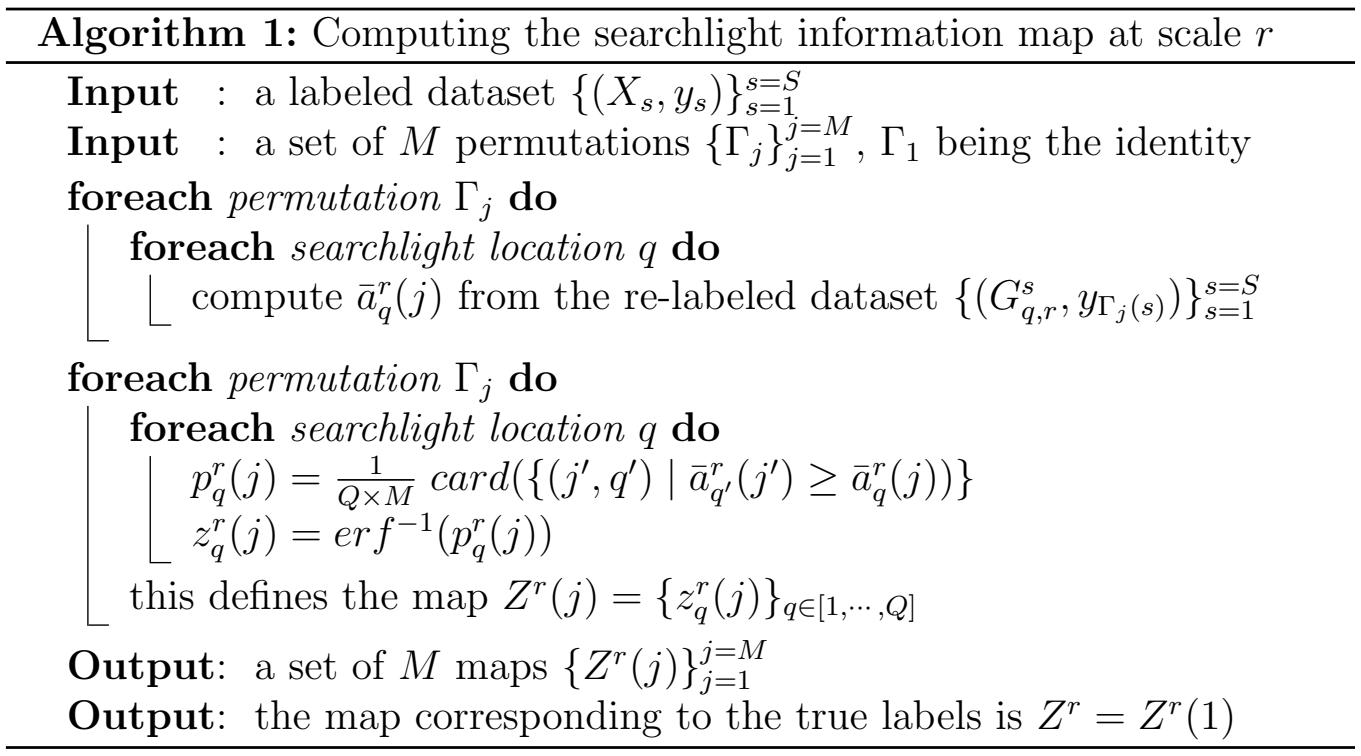

Note that we also compute the set of information maps $\left\{Z^{r}(j)\right\}_{j=1}^{j=M}$ obtained for all permutations because they will be used in the following. Also, by convention, the first permutation is set to be the identity, which means that the true information map $Z^{r}$ is included in this set as $Z^{r}(1)$.

\subsection{Multi-scale spatial inference}

In this section, we describe our third contribution, which is a multi-scale spatial inference strategy that both localizes the regions of the cortex where folding patterns are significantly different and estimates the spatial scale of these effects. First, we repeat the previously described searchlight procedure 
for a given set of $\rho$ scales $\mathfrak{R} \doteq\left\{r_{1}, \cdots, r_{\rho}\right\}$, yielding a set of information maps $\left\{Z^{r}\right\}_{r \in \Re}$, which each comes with its permuted versions $\left\{Z^{r}(j)\right\}_{j=1}^{j=M}$, forming a full set of $M \times \rho$ maps. Detecting at which locations $q$ and scales $r$ the classifier performance is above chance level - i.e testing $z_{q}^{r}>0, \forall(q, r)$ - is a massively univariate inference problem for which it is crucial to correct for multiple comparisons. Because these information maps exhibit correlations across both scales and space, an appealing way to deal with the multiple comparisons problem is to resort to cluster-based statistics, as introduced in Poline and Mazoyer (1993) and vastly used since in the neuroimaging literature. Following the non-parametric scheme described in Bullmore et al. $(1999)$, this can be done in three steps:

- applying an arbitrary threshold to a statistical map to form contiguous regions of supra-threshold locations, classically referred to as clusters;

- defining a cluster-wise statistic and computing it for each cluster;

- estimating the null distribution of the maximum cluster statistic using a set of maps obtained by permuting the labels of the observations, which provides corrected cluster-wise $p$-values (see Nichols and Holmes (2002)).

Thanks to the way our $z_{q}^{r}$ statistic was designed (see 2.4), the initial cluster-forming operation is easy to perform by setting a threshold $\tau$ that corresponds to the desired one-sided uncorrected point-wise $p$-value; for instance, thresholding at $z_{q}^{r}>\tau$ with $\tau=3.090$ corresponds to $p_{q}^{r}<0.001$. Note that because this is true for all values of $r$, a single threshold value can be used for all scales. Then, we need to select a cluster-wise statistic. The most commonly used one is the cluster size - the number of locations inside a cluster Poline and Mazoyer (1993), but it naturally favors large clusters. In order to avoid this bias, we use the cluster mass (Bullmore et al. (1999); Zhang et al. (2009)), i.e the sum of supra-threshold point-wise statistics, which should better preserve the chances of small clusters to be detected thanks to the high contrast of our $Z^{r}$ maps. Note that this potential benefit brought by using the cluster mass - instead of the cluster size - would have been lesser if we had directly used the accuracy as our summary statistic. Finally, we introduce two complementary strategies to estimate the empirical distribution of the cluster-wise statistic in this multi-scale setting. They are described in the following and summarized on Fig 4 . 


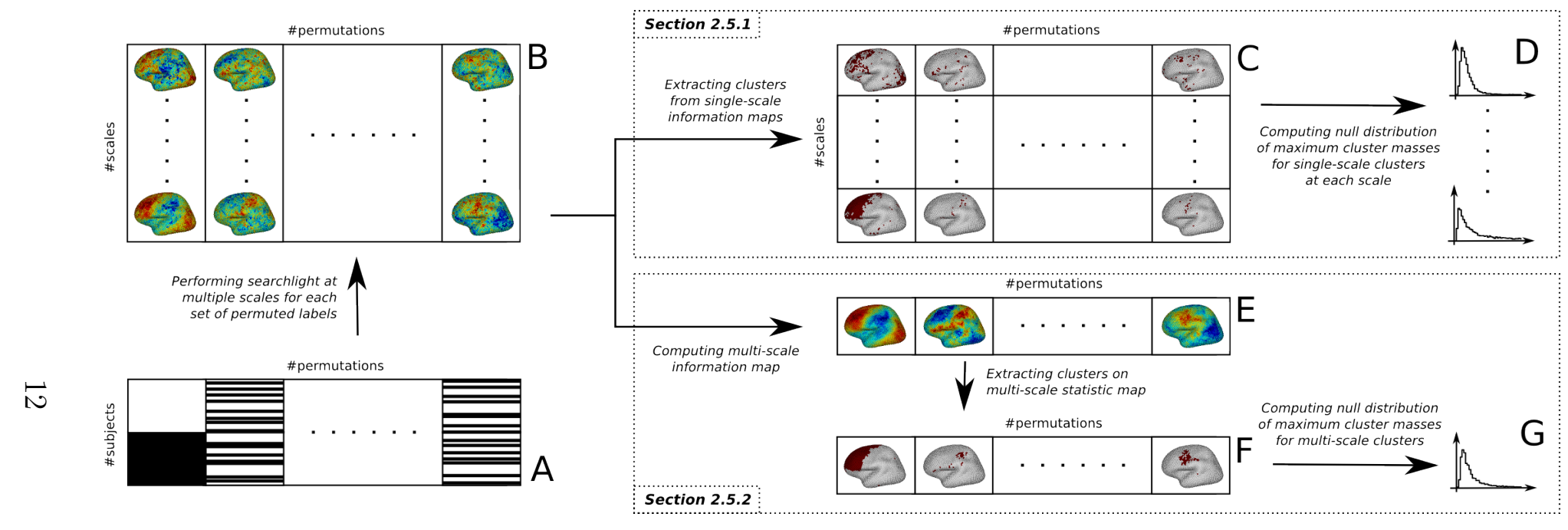

Figure 4: Illustrations of the two inference strategies. A: the set of permutations of the labels (each column is a permutation, black corresponds to $y=-1$ and white to $y=+1$; the first permutation in the left-most column contains the true labels). B: single-scale searchlight information maps, for each scale and each permutation. C \& D: single scale clusters, and the null distributions of their maximum cluster mass, estimated from the set of permuted single-scale clusters at each scale. E, F \& G: multi-scale information maps, multi-scale clusters, and the null distribution of their maximum cluster mass. The distribution shown in D and $\mathrm{G}$ are then used to assess the corrected $p$-values, respectively for the single-scale and the multi-scale clusters obtained with the true labels. 
2.5.1. Single-scale clusters with corrections for multiple comparisons across space and scales

The first strategy consists in defining clusters by independently thresholding each $Z^{r}$ map. We therefore designate these as single-scale clusters in the rest of the paper. The multi-scale nature of this first inference method hails from the correction for multiple comparisons, which is performed in two steps. First, we estimate the null distributions of the maximum cluster mass separately for each single scale, by pooling the masses of clusters obtained with all permutations at the considered scale (see Alg. 2). This yields $p$ values corrected for the multiple comparisons due to the repetition of tests in space. Note that it was necessary to distinguish null distributions between scales because they ended up being fairly different. In a second step, we used a Bonferroni correction to account for the repetition of tests across scales: multiplying the $p$-values obtained in the first step by the number of scales included in the analysis allows estimating corrected $p$-values that take into account the repetition of tests both in space and across scales, which can then be thresholded at the desired significance level to perform inference.

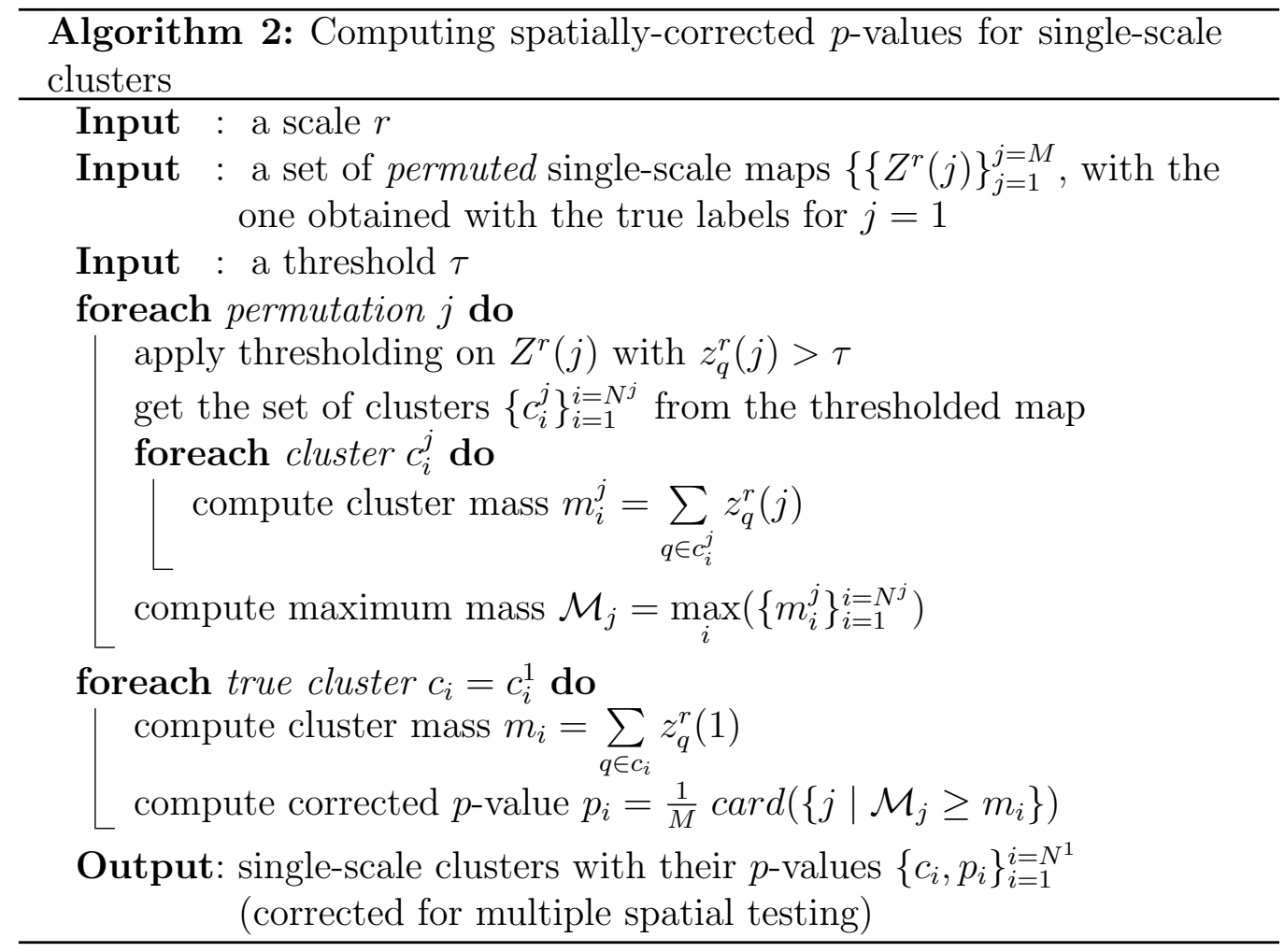


2.5.2. Multi-scale clusters with corrections for multiple comparisons across space

Our second strategy starts by designing a statistic that summarizes the results obtained across all scales at a given location $q$. Because we expect an effect to be observable across several consecutive scales, we first compute a sliding average of $z_{q}^{r}$ across scales. Then, in order to detect effects potentially present at any scale, we select the maximum value from the results of the sliding average operation. This gives

$$
\mathfrak{Z}_{q}^{R}=\max _{r \in \mathfrak{R}}\left(\frac{1}{R} \sum_{r^{\prime},\left|r-r^{\prime}\right| \leq R / 2} z_{q}^{r^{\prime}}\right),
$$

where $R$ represents the size of the averaging window used across scales. This results in a single multi-scale information map $\mathcal{Z}^{R}=\left\{\mathfrak{Z}_{q}^{R}\right\}_{q \in[1, \cdots, Q]}$, which summarizes the content of all single-scale maps $\left\{Z^{r}\right\}_{r \in \mathfrak{R}}$. We note $\left\{\mathcal{Z}^{R}(j)\right\}_{j=1}^{j=M}$ the set of corresponding permuted multi-scale maps. In the following, we will use the term multi-scale clusters to designate the clusters obtained by thresholding these multi-scale information maps. By changing the second input of Alg. 2 to this $\left\{\mathcal{Z}^{R}(j)\right\}_{j=1}^{j=M}$ set of permuted multi-scale maps, we can estimate the $p$-value - corrected for the repetition of tests performed in space - for each multi-scale cluster. Furthermore, at any location $q$ within a significant cluster, we can estimate the preferred scale of the effect as the value of $r$ that maximised the sliding average of $z_{q}^{r}$ across scales for the true labels:

$$
\hat{r}^{R}(q)=\arg \max _{r \in \Re}\left(\frac{1}{R} \sum_{r^{\prime},\left|r-r^{\prime}\right| \leq R / 2} z_{q}^{r^{\prime}}\right)
$$

\section{Experiments and results}

\subsection{Mapping gender and hemispheric differences}

In order to explore the capabilities of our framework, we study two classical brain morphometry problems: the mapping of gender differences and cortical asymmetries. Because some neurological disorders are expressed differently in males and females (see Ruigrok et al. (2014)) and others are associated with abnormal cortical lateralization Toga and Thompson (2003), studying these problems can directly provide information on the pathologies 
themselves. While they have already been studied using automatic morphometry methods such as VBM, SBM and OBM (see 3.3), our SGBM framework should shed a new light on both questions by examining differences in the organization of local folding patterns.

For this purpose, we use data from the Open Access Series of Imaging Studies ${ }^{2}$ (OASIS) cross-sectional database, which offers a collection of 416 subjects aged from 18 to 96 . For each subject, three to four individual T1-weighted MP-RAGE scans were obtained on a $1.5 \mathrm{~T}$ Vision system (Siemens, Erlangen, Germany) with the following protocol: in-plane resolution $=256 \times 256(1 \mathrm{~mm} \times 1 \mathrm{~mm})$, slice thickness $=1.25 \mathrm{~mm}, \mathrm{TR}=9.7 \mathrm{~ms}$, $\mathrm{TE}=4 \mathrm{~ms}$, flip angle $=10^{\circ}, \mathrm{TI}=20 \mathrm{~ms}$, TD $=200 \mathrm{~ms}$. Images were coregistered and averaged to create a single image with a high contrast-to-noise ratio. From this database, we selected two groups of 67 male and 67 female healthy right-handed subjects, aged 18 to 34 .

In practice, for the gender problem, we used the 134 subjects to set up a binary classification problem, with labels defined by $y=-1$ for a male subject and $y=+1$ for a female. For the asymmetry problem, an extra pre-processing step was carried out using the symmetric cortical template described in Greve et al. (2013), as used in Auzias et al. (2015) to study pit-based asymmetries. We then used the 134 symmetrized hemispheres of the male subjects to set up a binary classifcation problem where labels are $y=-1$ for a left hemisphere and $y=+1$ for a right hemisphere. For both problems, we use a Fibonacci point set of size $Q=2500$, which provides a dense enough sampling of the sphere (see Fig. 3) to observe the expected continuity in the information maps estimated by the searchlight scheme (see results hereafter). The number of permutations was set to $M=5000$, ensuring that the empirical distributions are accurately estimated. The crossvalidation used to estimate the average classification accuracy was carefully designed to ensure class balance in both the training and test datasets for each fold. As with any cluster-based analyses - which represent the vast majority of spatial inference methods used in neuroimaging, the initial clusterforming thresholding has an influence on the final results. With this in mind, we repeated our analysis with different threshold values and empirically selected the one that gave results which offered a good compromise between sensitivity and specificity. The results shown hereafter have been

\footnotetext{
${ }^{2}$ WWW. oasis-brains.org
} 
obtained with a cluster-forming threshold of $\tau=3.090$, which corresponds to a thresholding at $p<0.001$ on the uncorrected point-wise $p$-values associated with the classification score. Finally, we used the following set of scales in order to study the multi-scale properties of local sulcal patterns: $\mathfrak{R} \doteq\{30,35,40,45,50,55,60,65,70,75,80,85,90 \mathrm{~mm}\}$. Each scale value defines the radius of a searchlight spherical neighborhood that is computed on the freesurfer sphere, i.e. the common space for all subjects, which has a $100 \mathrm{~mm}$ radius. The smallest neighborhood (30 $\mathrm{mm}$ Euclidean radius) is a spherical cap whose surface area is $2.25 \%$ of the sphere surface area. Equivalently, the largest neighborhood (90mm scale) represents $20.75 \%$ of the sphere area. In other words, we use a scale range that varies between $2.25 \%$ and $20.75 \%$ of an hemisphere surface area. This will yield patterns between the size of a small gyrus and one of a brain lobe, thus covering the smallest (Im et al., 2010; Auzias et al., 2015) and largest (Im et al., 2011, 2012) effects previously reported in the literature when studying sulcal pits.

\subsection{Results: methodological considerations}

In this section, all the maps presented follow the same format. Each of the elements of the spherical Fibonacci point set is represented by a small sphere and projected onto the inflated cortical mesh. This allows for an easy visualization of the global maps.

\subsubsection{Examining raw information maps}

In order to assess the results provided by our SGBM framework, we first qualitatively observe raw single-scale and multi-scale information maps obtained with our pit-based searchlight scheme.

Single-scale maps $Z^{r}$ are shown on Fig. 5. They display some contrast at all scales $r$, i.e they contain locations of low and high $z_{q}^{r}$-values, sometimes reaching $z_{q}^{r}=5$ or more - which corresponds to very significant uncorrected point-wise $p$-values $\left(p_{q}^{r}<10^{-6}\right)$. We can clearly observe the spatial correlation through the fact that the points of high statistic values are grouped together. Furthermore, the spatial smoothness of the maps and the size of the groups of searchlight locations with high $z_{q}^{r}$-values increase with $r$, which is a typical behavior of searchlight information maps Kriegeskorte et al. (2006). Finally, the persistence of the regions with high $z_{q}^{r}$-values across several consecutive values of $r$ attests of the correlation across scales of the $Z^{r}$ maps, which also supports the relevance of the construction scheme used to define the multi-scale statistic $\mathfrak{Z}_{q}^{R}$ (see Eq. 6). 


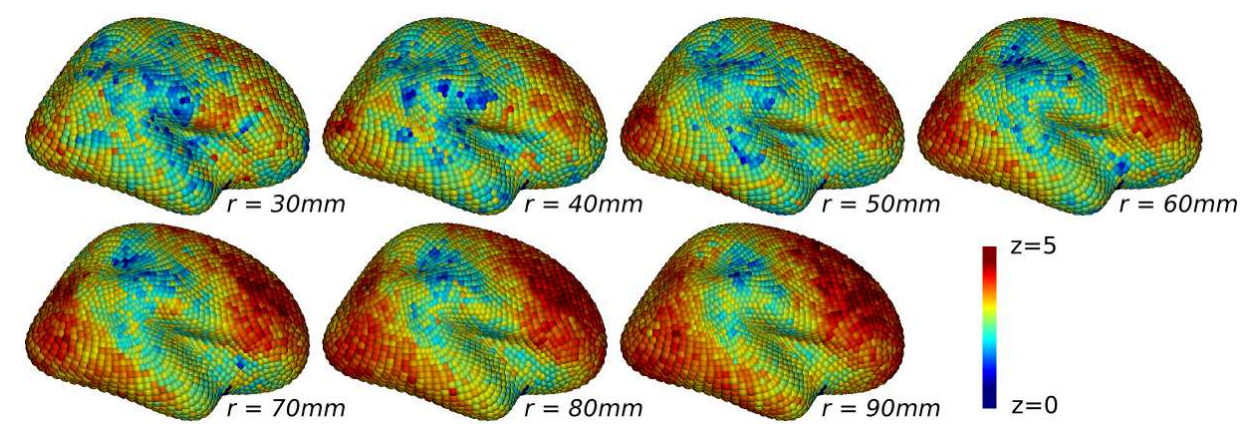

Figure 5: Examples of raw single-scale information maps $Z^{r}$ for different neighborhood radiuses $r$ (gender differences, external face of the right hemisphere).

We now examine the multi-scale maps $\mathcal{Z}^{R}$, displayed on Fig. 6. Each of them summarizes the information contained at all scales $r \in \mathfrak{R}$. For $R=1$, $\mathfrak{Z}_{q}^{R}$ is the max of all $z_{q}^{r}$ across all scales $r$; for $R=\operatorname{card}(\mathfrak{R})$, i.e for $R=13$ in our experiments, $\mathfrak{Z}_{q}^{R}$ is the average of $z_{q}^{r}$ across all scales. Overall, these maps are smoother than the single-scale $Z^{r}$ maps, which is expected because the construction of the multi-scale statistic $\mathfrak{Z}_{q}^{R}$ acts as a smoothing operation across scales. For the smaller values of $R$, the contrast of the statistic map is weaker than the one offered by single-scale maps: some very large portions of the cortex show high values of $\mathfrak{Z}_{q}^{R}$, which means that the specificity offered by low values of $R$ is limited. For very large values of $R$, the maps contain less locations with high statistical values, which is also expected because in this case, a high $\mathfrak{Z}_{q}^{R}$-value reflects a phenomena that exist over a larger number of consecutive scales. Therefore, it seems adequate to use an intermediary value of $R$, for which $\mathfrak{Z}_{q}^{R}$ averages $z_{q}^{r}$ over a limited number of consecutive scales.

\subsubsection{Qualitative assessment of single-scale clusters}

We then examine the single-scale clusters. Figs. 7 and 8 show the clusters which present a $p$-value lower than 0.05 , after correction for multiple comparisons across space and scales with the method described in Section 2.5.1. For both the gender and asymmetry problems, our framework detects significant clusters at all scales with $r$ at least $40 \mathrm{~mm}$, demonstrating its detection power. Almost all clusters are persistent across scales, i.e for a given cluster at scale $r$, there exist clusters at nearby cortical locations for nearby scales. The clusters detected at finer scales are overall smaller than the ones 


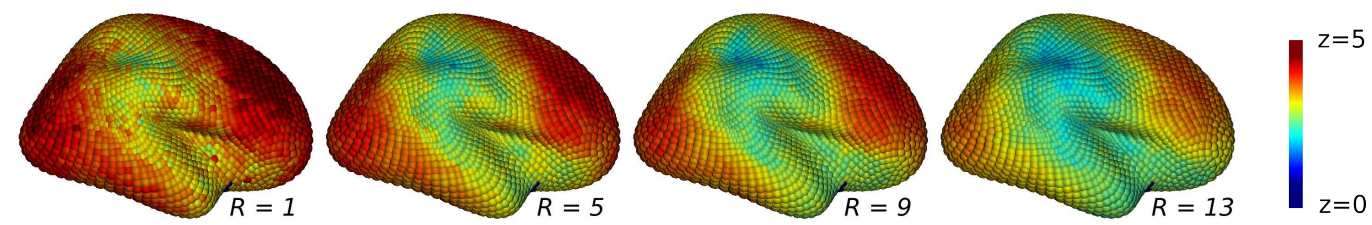

Figure 6: Examples of raw multi-scale information maps $\mathcal{Z}^{R}$ (gender differences, external face of the right hemisphere). Each of these maps summarizes the information from all single-scale $Z^{r}$ maps shown on Fig 5 . This multi-scale statistic depends on the number $R$ of consecutive scales $r$ over which the single-scale statistic is averaged (see Eq. 6).

detected with larger window sizes. This is caused by the larger smoothing effect of the large window sizes (as previously observed on the raw maps, see on Fig. 5); furthermore, we noted that the critical cluster mass corresponding to a corrected $p$-value of 0.05 (estimated by the procedure described in Section 2.5.1) increases with the size of the window, which therefore discards the smallest clusters at large scales. However, some small clusters remain significant at large scales. This overall demonstrates that our cluster-based spatial inference strategy is effective; in particular, the use of the cluster mass associated with our $z_{q}^{r}$ statistic makes it possible to detect clusters at fine spatial scale and small clusters at larger scales, which is known to be challenging in practice in such a multi-scale context.

\subsubsection{Qualitative assessment of multi-scale clusters}

Figs. 9 and 10 show the significant clusters (corrected $p<0.05$ ) obtained with the method described in 2.5.2, for $R \in\{1,3,5,7,9,11,13\}$. The observations made previously on the raw statistic map gets confirmed: some overly large clusters are detected for the smaller values of $R$, while nothing is detected for larger values $(R \geq 11)$. This confirms that an intermediary value of $R$ might offer a satisfactory compromise between specificity and sensitivity. This is concordant with the intuition that an effect can live across several consecutive scales, but not over a very large number of consecutive scales as sought after with large $R$ values.

\subsubsection{Comparing single-scale and multi-scale results}

We will now compare the clusters detected by our single-scale and multiscale approaches. Our objective is two-fold: i) to assess the consistency, or lack thereof, of the results of the two approaches, and ii) to facilitate the 


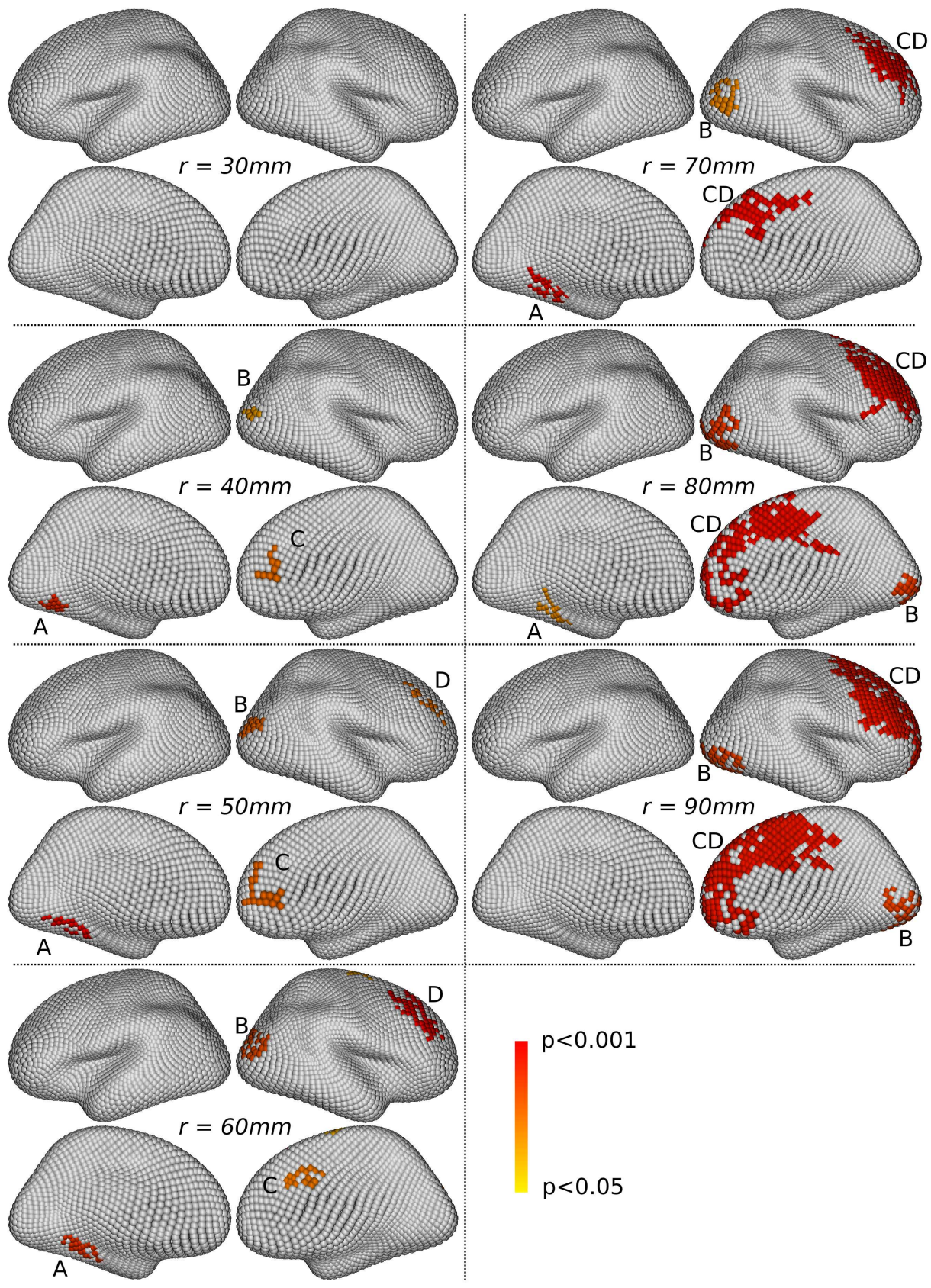

Figure 7: Gender differences. For each value of $r$, four views are shown, two per hemisphere: the left hemisphere is shown on the left, and the right on the right, with respectively their external and internal faces at the top and the bottom. The significant single-scale clusters (corrected $p<0.05$ ) are colored with their $p$-value. Four main clusters, tagged A, B, C and D, appear to be persistent across scales, sometimes after having merged. 


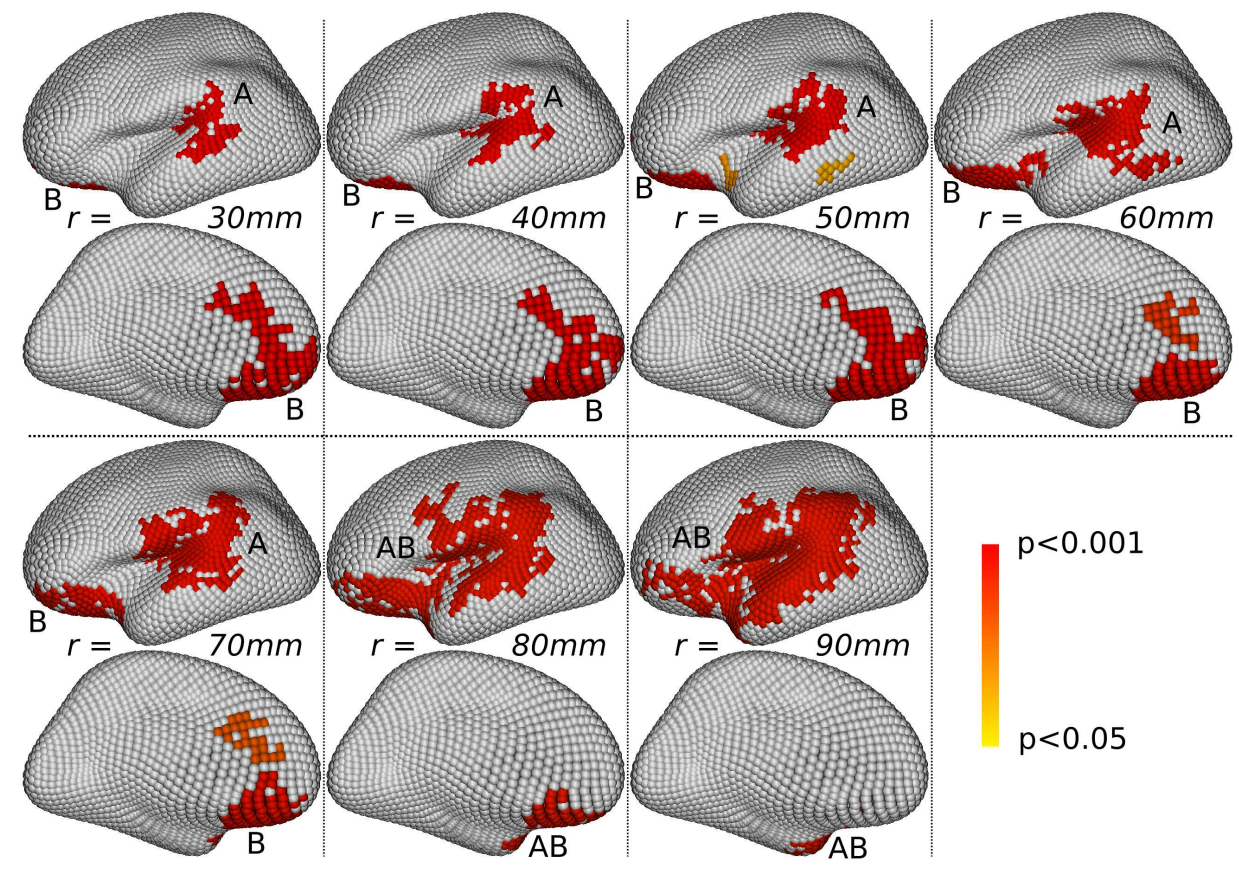

Figure 8: Asymmetries. For each value of $r$, the single-scale clusters presenting significant differences between the left and right hemispheres (corrected $p<0.05$ ) are shown on the external and internal faces of the left hemisphere. Two main clusters, tagged A and B, appear to be persistent across scales. 


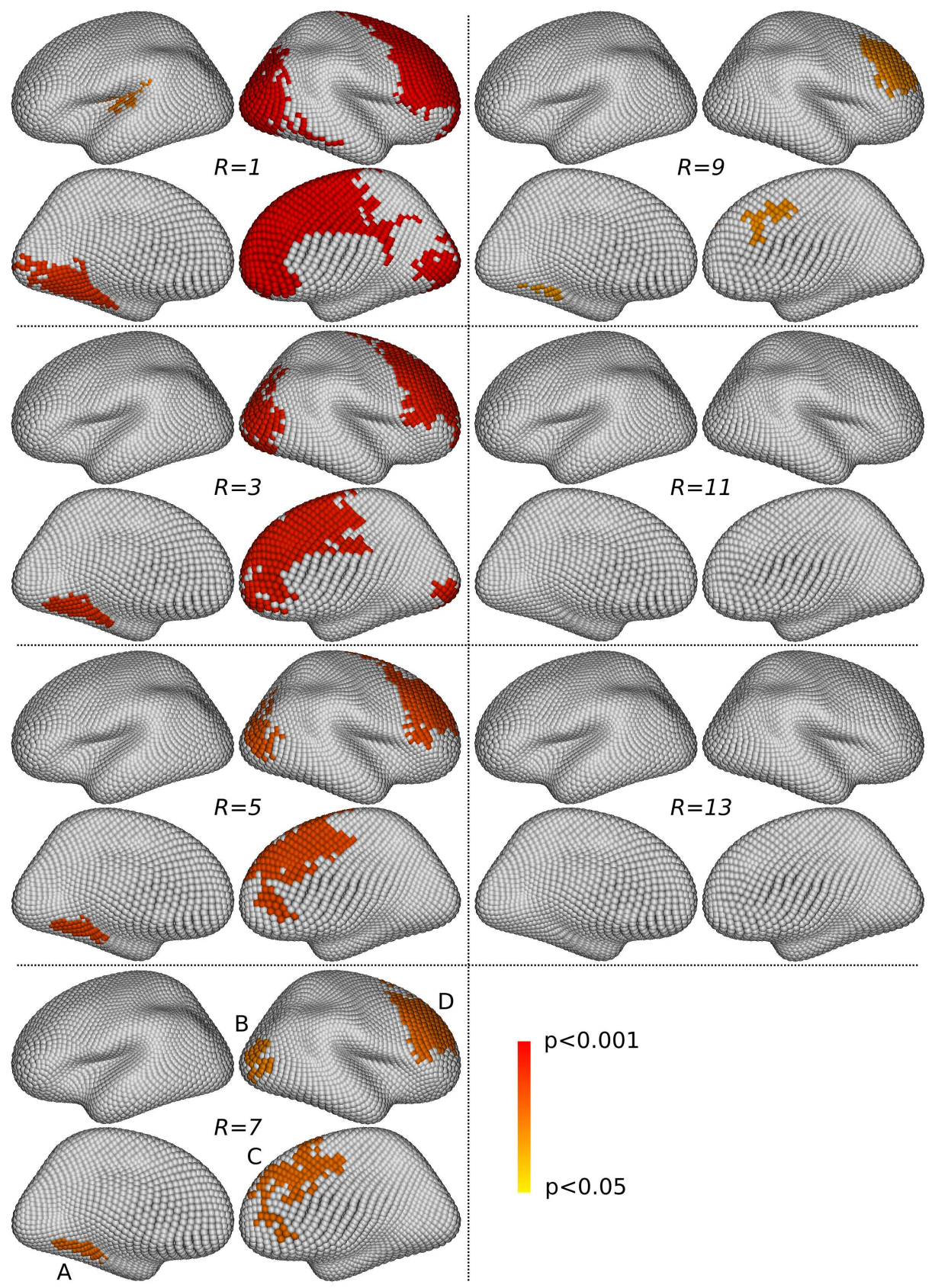

Figure 9: Gender differences. Simlarly to Fig,7, four views are presented for each value of $R$, showing thresholded significant multi-scale clusters (corrected $p<0.05$ ). For $R=7$, four clusters survive, at similar locations as single-scale ones; they are tagged A, B, C and D, accordingly to Fig. 7 


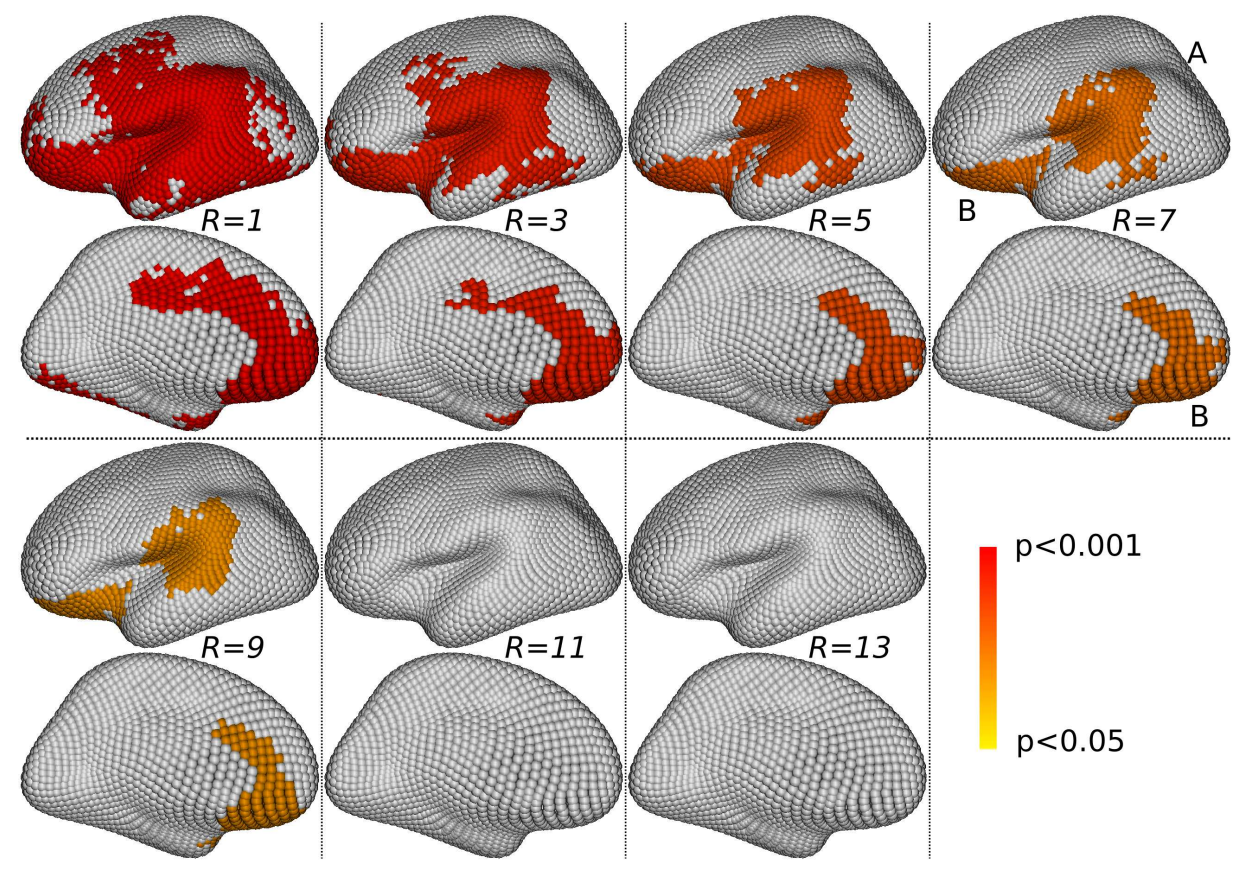

Figure 10: Asymmetries. Significant muli-scale thresholded clusters. For $R=7$, the same two clusters as in the single-scale analysis are detected; they are tagged A and B, accordingly to Fig. 8 . 
selection of the value of the $R$ parameter of our multi-scale statistic.

For the gender problem, we examine Fig. 7 (single-scale clusters) and Fig. 9 (multi-scale clusters). The single-scale approach detects clusters in four main locations (tagged A, B, C and D on Fig. 7), which are present across a large number of scales. Their precise location and extent vary smoothly across scales. For instance, the cluster at location B slightly moves forward when the scale $r$ increases; some merging phenomena also happen between clusters, as with the clusters at locations $\mathrm{C}$ and $\mathrm{D}$, which are separated at scales $r \leq 60 \mathrm{~mm}$ and fused into one larger cluster at scales $r \geq 70 \mathrm{~mm}$. The multi-scale approach detects clusters in similar locations for various values of the $R$ parameter; however, the results with $R=7$ are the only ones for which there are four separated clusters at the four locations A, B, C and D.

For the asymmetry problem, we examine Fig. 8 (single-scale clusters) and Fig. 10 (multi-scale clusters). The single-scale approach detects clusters in two main locations (tagged $\mathrm{A}$ and $\mathrm{B}$ on Fig. 8), which are present across almost the full range of scales from $r=30 \mathrm{~mm}$ to $r=90 \mathrm{~mm}$. The clusters present around these locations also fuse together for $r \geq 80 \mathrm{~mm}$. The multiscale approach detects clusters in similar locations for various values of the $R$ parameter; however, only the results obtained with $R=7$ and $R=9$ provide two separated clusters for each location A and B.

Overall, both our single- and multi-scale inference strategies offer satisfactory detection power. The fact that the significant clusters of both approaches occupy similar locations clearly demonstrates their consistency. In order to choose the value of the parameter $R$ of the multi-scale strategy, we showed that choosing an intermediary value provides a good compromise between sensitivity and specificity, as found in our restults with $R=7$ for the gender differences and asymmetry problems studied here.

\subsection{Results: neuroscience considerations}

In this section, we examine the neuroscientific relevance of the results obtained with our SGBM framework for the two problems of gender differences and cortical asymmetries, based on the multi-scale clusters detected with $R=7$. Each map shown on Figs. 11 and 12 presents the set of searchlight locations (i.e the Fibonacci points displayed on the sphere on Fig. 3) included in a given significant cluster and projected onto the folded cortical mesh. This allows for a precise localization of the extent of each cluster on the cortex which can facilitate the interpretation. 


\subsubsection{Gender differences}

Gender differences have mostly been studied using standard morphometry tools such as VBM, with notably a meta-analysis presented in Ruigrok et al. (2014) and SBM, for instance in Im et al. (2006); Lv et al. (2010)). To our knowledge, the only sulcus-based study that used OBM is Duchesnay et al. (2007).

Our SGBM framework detected four clusters that contain the center locations of searchlight windows in which the patterns of sulcal pits differ between genders, which are shown on Fig. 11. Cluster A is centered in the collateral sulcus of the left hemisphere. It is a fairly small cluster comprising 35 searchlight points, with an average preferred scale of $\hat{r}=60 \mathrm{~mm}$ (following the definition of $\hat{r}$ in 2.5.2). Its localization is consistent with regions that were significant in Ruigrok et al. (2014); Im et al. (2010); Lv et al. (2010). Cluster B is located in the right hemisphere, centered around the lateral occipital sulcus. It comprises 29 searchlight locations, has an average preferred scale of $\hat{r}=65 \mathrm{~mm}$, and is concordant with very small spots appearing in the surface based studies only (Im et al. (2010); Lv et al. (2010)). Moreover, the depth difference observed in the lingual sulcus in Duchesnay et al. (2007) probably contributes to the effect detected in this cluster - which, in its single scale version at $r=80 \mathrm{~mm}$, includes the lingual sulcus on the internal face (see Fig. 7). Cluster C, located in the right hemisphere, has its center of mass in the cingulate gyrus. It is a large cluster with 98 searchlight locations with an average preferred scale of $\hat{r}=75 \mathrm{~mm}$. Finally, cluster $\mathrm{D}$ is a large cluster centered in the middle frontal gyrus, comprising 130 searchlight locations and with an average preferred scale of $\hat{r}=75 \mathrm{~mm}$. Small regions located within the large clusters $\mathrm{C}$ and $\mathrm{D}$ were also found significant in Ruigrok et al. (2014); Im et al. (2010); Lv et al. (2010), demonstrating the overall consistency of our results with the literature.

\subsubsection{Asymmetries}

Cortical asymmetries have been studied using a large variety of methods, from VBM (see Good et al. (2001) for instance) and SBM (in Van Essen et al. (2012)) to OBM (see Duchesnay et al. (2007)) and sulcal pits characterization (in Im et al. (2010); Auzias et al. (2015)).

By studying the organization of local patterns of sulcal pits, our SGBM framework was able to detect two clusters containing the center locations of searchlight windows in which cortical folding patterns present local asymmetries, which are shown on Fig. 12. The first one (Cluster A) is a large 


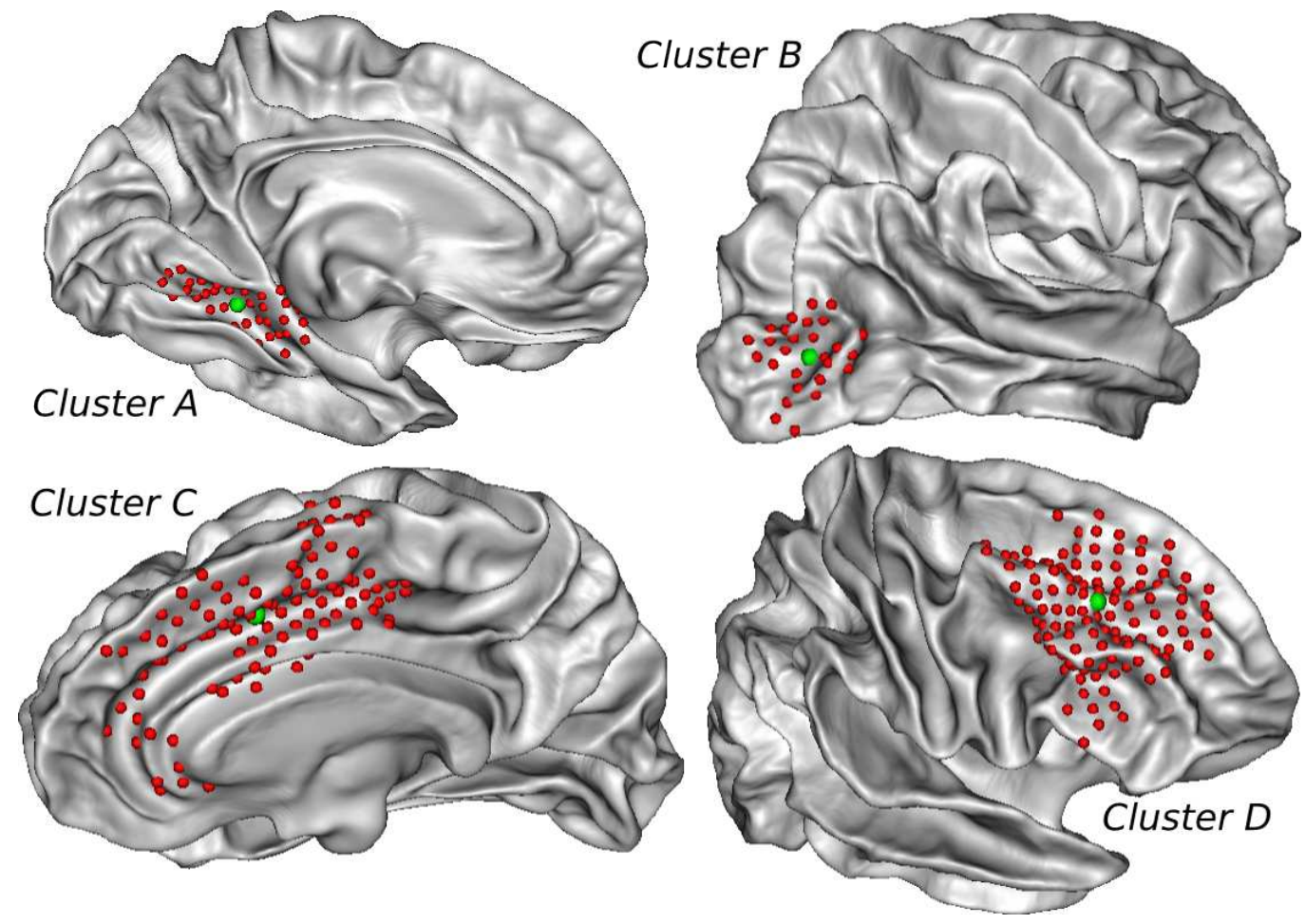

Figure 11: Gender clusters. Each map presents the Fibonacci points included in a given cluster, among the four significant clusters detected using our multi-scale statistic $\mathfrak{Z}^{R}$ and $R=7$. The larger green sphere represents the center of mass of the cluster. 

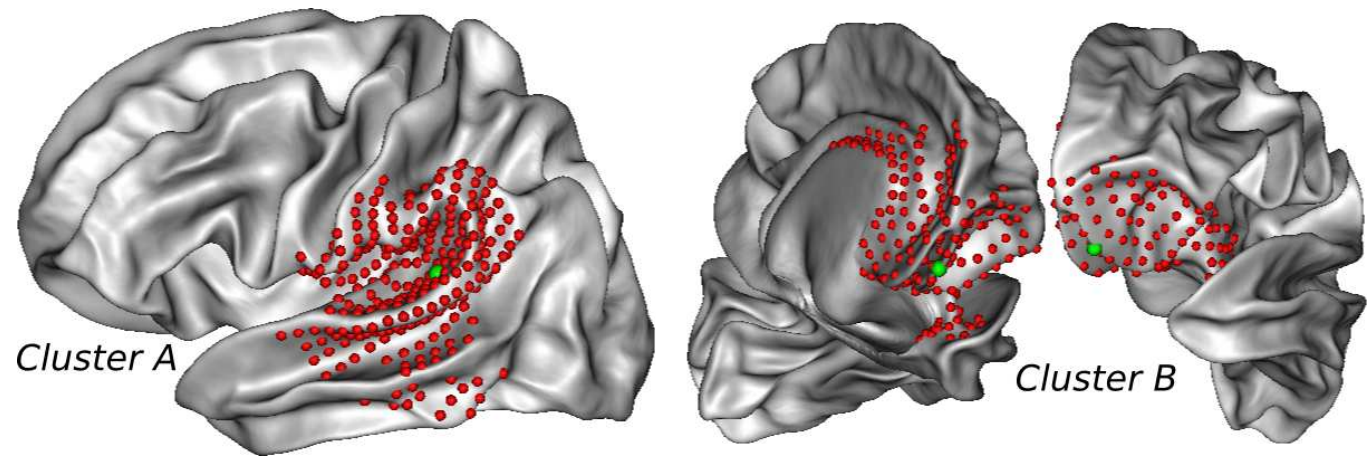

Figure 12: Asymmetry clusters. These maps each present one of the two significant clusters detected using our multi-scale statistic $\mathfrak{Z}^{R}$ with $R=7$. The larger green sphere represents the center of mass of the cluster. For clarity, the map of Cluster B (right) is presented under two different views.

cluster comprising 295 searchlight locations, with an average preferred scale of $\hat{r}=70 \mathrm{~mm}$, which is centered around the planum temporale and also encompasses the posterior part of the sylvian fissure, the supra-marginal gyrus and some parts of the superior temporal gyrus and sulcus. These regions are all individually reported in the literature dedicated to cortical asymmetries - Van Essen et al. (2012); Im et al. (2010); Auzias et al. (2015), all using univariate analyses, and Duchesnay et al. (2007), which found a depth difference in the superior temporal sulcus. This shows that the multivariate nature of our analysis allows to detect such locally distributed effects among a complex pattern of sulcal basins. Cluster B comprises 205 searchlight locations centered around the frontal pole and presents an average preferred scale of $\hat{r}=60 \mathrm{~mm}$. It includes part of the lateral prefrontal cortex, and extends on the internal face to the middle part of the cingulate sulcus. These regions have been consistently detected with morphometry approaches such as VBM (Good et al. (2001)) and also with the study of sulcal pits frequency described in Auzias et al. (2015) - which used the same pit extraction algorithm as ours. These concordant results corresponds to the frontal petalia, a protrusion of the anterior part of left hemisphere towards the right one Toga and Thompson (2003). 


\section{Discussion}

We have introduced Structural Graph-Based Morphometry (SGBM), a new fully automatic brain morphometry framework based on a multi-scale searchlight scheme that enables to localize differences in graphs of sulcal pits between populations. This is the first time that a searchlight scheme is designed for anatomical MR images of the brain and embedded in a multi-scale inference strategy. The main strengths of our framework are i) to capitalize on structural pattern recognition tools in order to discriminate complex cortical folding patterns represented as graphs of sulcal pits, ii) to overcome the intrinsic limitations of approaches that focus on a pre-defined region thanks to the searchlight approach, iii) to embed this searchlight approach into a powerful cluster-based multi-scale inference strategy.

The results obtained in this paper demonstrate the detection power of our SGBM framework in order to localize regions where sulcal pit-graphs are significantly different between groups, thus showing its ability to overcome the intrinsic limitations of previous ROI-based approaches. The multi-scale character of our SGBM framework was called for by the lack of a priori knowledge on the size of the relevant patterns. The multi-scale nature of these effects is clearly confirmed by our results: on the one hand, single-scale clusters are detected over a large span of scales, with varying locations and sizes (see Figs. 7 and 8), and on the other hand, the detection of multi-scale clusters (see Figs. 9 and 10 ) shows that these effects are present over several consecutive scales. These multi-scale clusters also come with an estimate of the preferred scale of the effect, which vary between 60 and $75 \mathrm{~mm}$ for the studied problems. These values, which are defined in freesurfer's common spherical space, correspond to intermediary region sizes between the smallest and largest previously reported effects, i.e to folding patterns comprising several sulci. This confirms that local interactions between neighboring sulcal basins or sulci can be informative. Moreover, our two inference strategies showed their complementarity. The multi-scale scheme is computationally efficient and offers a compact representation of the results, summarized in a single map, which eases the interpretation of the results. Comparatively, the single-scale clusters provide a more detailed scale by scale description of the phenomena at hand, but come at a higher computational cost - because the number of permutations to perform is multiplied by the number of scales considered. And because of the large number of resulting maps - one per scale, the results of the single-scale scheme might be more difficult to in- 
terpret. Future work could address this latter drawback by modelling more precisely the relations between clusters obtained at different scales, as was done in Operto et al. (2012) for fMRI data. Finally, note that our multi-scale inference strategy could benefit to other searchlight applications, in particular for fMRI data analysis where it has grew very popular; indeed, Etzel et al. (2013) suggested to use a systematic multi-scale approach to provide more robust statistical results and thus improve the interpretability, and our method directly fullfills this need.

The fact that the results obtained with our SGBM framework are in line with the existing literature also validate the non linear Support Vector Classifier as an adequate statistical model to study patterns of sulcal pits and their differences between populations. This demonstrates that the graph kernel that we introduced in 2.3 is a valid similarity measure for comparing pit-graphs, thus providing an alternative to the one of $\operatorname{Im}$ et al. (2011). It is to be noted that the choice of the weights that define Im's metric required a set of dedicated experiments (see Im et al. (2011)) and the result of their followup work (Im et al. $(2012,2015))$ heavily depends on the values of these seven hyper-parameters. In contrast, our kernel only has two hyper-parameters that we directly estimate from the data. The only available element of comparison between the two measures is their ability to detect gender differences. In Im et al. (2012), the authors reported no gender differences in their control group (first sentence in Results, p.3011), whereas our technique was able to find several regions with highly significant differences between male and female subjects. These differences can be explained by several reasons: i) our dataset was much larger (134 vs. 26 subjects); ii) our pit detection algorithm is slightly different; iii) their study focused on predetermined large regions (brain lobes), whereas our framework automatically estimates the localization and the size of the region; iv) our inference strategy is more powerful, thanks to the exploitation of the local redundancy that exist between locations within a cluster. In theory, it is also possible that our kernel provides a more expressive graph similarity measure, but we have no element to concur with this possibility. On the contrary, it was shown - respectively in Im et al. (2010) and in Takerkart et al. (2014) (where a kernel with a similar design was used on fMRI patterns) - that both similarity measures effectively use all elements of the graphs - their structure, the location of the graph nodes and their attributes. We therefore believe that they are sensitive to the same features of the folding pattern, that include: 
- differences in sulcal depth distributed across a local folding pattern, as suggested by the concordant results with Duchesnay et al. (2007) which detected depth differences co-localized with some of the SGBM clusters,

- differences in the local number of pits, as showed by the consistent results of SGBM and the pit frequency study of Auzias et al. (2015),

- differences in pits localization, as suggested by the agreement between our results and the one of Im et al. (2010) which examined pits displacements;

- local organization differences, which might be induced by the two previous types of effects.

When it comes to interpreting the results provided by our SGBM framework, we demonstrated that a visualization tool such as the one used in 3.3 allows to easily localize the detected clusters on the cortex, which makes it possible to directly compare SGBM with other automatic morphometry methods such as VBM, SBM and OBM. For the gender differences and asymmetry problems, our results are consistent with previously reported differences in grey matter volume (VBM), cortical thickness (SBM) and sulcal shape (OBM), which was expected because these different features are - at a certain level - linked together. But for the first time, SGBM makes it possible to automatically detect markers associated with the spatial organization of cortical folding. To go beyond a simple reporting of the localization of the detected clusters and in order to unveil the neuroscientific underpinnings of such effect, there is a crucial need to understand which of the four aforementioned characteristics of the folding patterns actually contributes to the group discrimination in a given cluster. This is a very challenging problem because of the complex nature of cortical folding and because our graph kernel combines several feature types in a single similarity measure. But its modular design makes it possible to conduct posthoc sensitivity analyses similarly to what was done in Im et al. (2011, 2012, 2015) and in Takerkart et al. (2014), which simply consists in adding or removing a feature, repeating the analysis and assessing its contribution by examining the performance difference. Finally, because our similarity measure is a positive definite kernel, we can exploit the vast array of available kernel methods to perform other types of posthoc analyses that could help us gain further neuroscientific insights. 
For instance, we could perform dimensionality reduction using kernel PCA in order to examine the dimensions in graph-space that explain the largest variance in a given cluster. Then, similarly to what was done in Sun et al. (2012a) using another dimensionality reduction technique, it is possible to project the pit-graphs of all subjects along each principal dimension and to plot them along the corresponding axis in order to visually assess the main factor(s) of influence. Beyond these two leads - sensitivity analyses and dimentionality reduction, the intrinsic complexity of the folding pattern leads us to believe that further work will be needed to better understand what contributes to the differences detected with our SGBM framework and how.

\section{Conclusion}

We have introduced a new brain mapping technique dedicated to studying differences in the local organization of cortical anatomy, by the mean of patterns constructed from the deepest part of the cortical sulci - the sulcal pits. Our technique - that we called Structural Graph-Based Morphometry (SGBM), is the first morphometry framework that combines a graph kernel to compare sulcal pit-graphs, a searchlight scheme to overcome the limitations of ROI-based approaches and a multi-scale inference strategy that allows to find significant effects at different spatial scales. We have demonstrated that SGBM offers good detection power on two problems that respectively examined the anatomical gender differences and the cortical asymmetries. This versatile and powerful pit-based morphometry framework should therefore find numerous applications, in particular to study cortical development and clinical populations, two fields where examining the organization of sulcal patterns at different spatial scales is particularly relevant.

\section{Acknowledgments}

We would like to thank the anonymous reviewers for their constructive suggestions and comments. G. Auzias and L. Brun were funded through research grants from Fondation de France (OTP 38872) and Fondation Orange (S1 2013-050). The research of S. Takerkart and O. Coulon is supported by the French Centre National de la Recherche Scientifique. 


\section{Vitae}

Sylvain Takerkart is a research engineer at the French Centre National de la Recherche Scientifique (CNRS), working as the manager of the scientific computing facility of the Institut de Neurosciences de la Timone. His research activities focus on developing and applying machine learning tools for new image processing problems encountered in neuroscience.

Guillaume Auzias received the Masters degree in Applied Mathematics from University Pierre and Marie Curie - Paris 6 in 2006 and his PhD at the Brain and Spine (ICM) institute in 2009. The main goal of his work is to demonstrate the benefit of integrating information derived from cortical folds in order to define correspondences between subjects.

Lucile Brun is a research engineer at the Institut de Neurosciences de la Timone. With a dual qualification in medical imaging and neuroscience, she is a member of the Social Cognition across Lifespan and Pathologies research group, which studies morphology changes in neuro-developmental pathologies. She contributes to the design of extraction methods for new anatomical features such as the sulcal pits.

Olivier Coulon is a director of research at the Laboratoire des Sciences de l'Information et des Systèmes, CNRS, Aix-Marseille University, and the head of the Methods and Computational Anatomy research group (http: //www.meca-brain.org). For more than 15 years his research has been dedicated to neuroimaging data analysis. He is particularly interested in modelling and quantifying cortical organization and variability, and the link between anatomy and function.

\section{References}

Aizerman, M., Braverman, E., Rozonoer, L., 1964. Theoretical foundations of the potential function method in pattern recognition learning. Automation and Remote Control 25, $821-837$.

Ashburner, J., Friston, K.J., 2000. Voxel-based morphometry-the methods. NeuroImage 11, 805-821. URL: http://linkinghub.elsevier.com/ retrieve/pii/S1053811900905822, doi:10.1006/nimg.2000.0582.

Auzias, G., Brun, L., Deruelle, C., Coulon, O., 2015. Deep sulcal landmarks: Algorithmic and conceptual improvements in the definition and extraction of sulcal pits. NeuroImage 111, 12-25. URL: http:// 
linkinghub.elsevier.com/retrieve/pii/S1053811915001007, doi:10. 1016/j.neuroimage.2015.02.008.

Auzias, G., Viellard, M., Takerkart, S., Villeneuve, N., Poinso, F., Da Fonséca, D., Girard, N., Deruelle, C., 2014. Atypical sulcal anatomy in young children with autism spectrum disorder. NeuroImage: Clinical 4, 593-603. URL: http://linkinghub.elsevier.com/retrieve/pii/ S2213158214000382, doi:10.1016/j.nicl.2014.03.008.

Boucher, M., Whitesides, S., Evans, A., 2009. Depth potential function for folding pattern representation, registration and analysis. Medical image analysis 13, 203-14. URL: http://www.ncbi.nlm.nih.gov/pubmed/ 18996043 .

Bullmore, E.T., Suckling, J., Overmeyer, S., Rabe-Hesketh, S., Taylor, E., Brammer, M.J., 1999. Global, voxel, and cluster tests, by theory and permutation, for a difference between two groups of structural MR images of the brain. Medical Imaging, IEEE Transactions on 18, 32-42. URL: http://ieeexplore. ieee.org/xpls/abs_all.jsp?arnumber $=750253$.

Cachia, A., Paillère-Martinot, M.L., Galinowski, A., Januel, D., de Beaurepaire, R., Bellivier, F., Artiges, E., Andoh, J., Bartrés-Faz, D., Duchesnay, E., Rivière, D., Plaze, M., Mangin, J.F., Martinot, J.L., 2008. Cortical folding abnormalities in schizophrenia patients with resistant auditory hallucinations. NeuroImage 39, 927-935. URL: http:// linkinghub.elsevier.com/retrieve/pii/S1053811907007720, doi:10. 1016/j.neuroimage.2007.08.049.

Coulon, O., Mangin, J.F., Poline, J.B., Zilbovicius, M., Roumenov, D., Samson, Y., Frouin, V., Bloch, I., 2000. Structural group analysis of functional activation maps. NeuroImage 11, 767-782. URL: http:// linkinghub.elsevier.com/retrieve/pii/S1053811900905809, doi:10. 1006/nimg.2000.0580.

Dale, A., Fischl, B., Sereno, M.I., 1999. Cortical surface-based analysis: I. segmentation and surface reconstruction. NeuroImage 9, 179 - 194.

Duchesnay, E., Cachia, A., Roche, A., Rivière, D., Cointepas, Y., Papadopoulos-Orfanos, D., Zilbovicius, M., Martinot, J.L., Régis, J., Mangin, J.F., 2007. Classification based on cortical folding patterns. IEEE Trans. Med. Imaging 26, 553-565. 
Etzel, J.A., Zacks, J.M., Braver, T.S., 2013. Searchlight analysis: Promise, pitfalls, and potential. NeuroImage 78, 261-269. URL: http:// linkinghub.elsevier.com/retrieve/pii/S1053811913002917, doi:10. 1016/j.neuroimage.2013.03.041.

Fischl, B., Sereno, M.I., Tootell, R.B., Dale, A.M., others, 1999. High-resolution intersubject averaging and a coordinate system for the cortical surface. Human brain mapping 8, 272284. URL: http://www.researchgate.net/profile/Anders_Dale/ publication/230865729_High-resolution_intersubject_averaging_ and_a_coordinate_system_for_the_cortical_surface/links/ 00b49516505b8ea19a000000.pdf.

Gärtner, T., Flach, P.A., Wrobel, S., 2003. On graph kernels: Hardness results and efficient alternatives, in: Proc. of the 16th Conf. on Computational Learning Theory.

Good, C.D., Johnsrude, I., Ashburner, J., Henson, R.N., Friston, K.J., Frackowiak, R.S., 2001. Cerebral asymmetry and the effects of sex and handedness on brain structure: A voxel-based morphometric analysis of 465 normal adult human brains. NeuroImage 14, 685-700. URL: http:// linkinghub.elsevier.com/retrieve/pii/S1053811901908572, doi:10. 1006/nimg. 2001.0857.

Greve, D.N., Van der Haegen, L., Cai, Q., Stufflebeam, S., Sabuncu, M.R., Fischl, B., Brysbaert, M., 2013. A surface-based analysis of language lateralization and cortical asymmetry. Journal of Cognitive Neuroscience 25, 1477-1492. URL: http://www.mitpress journals.org/doi/abs/10. 1162/jocn_a_00405, doi:10.1162/jocn_a_00405.

Haussler, D., 1999. Convolution kernels on discrete structures. Technical Report UCSC-CRL-99-10. UC Santa Cruz.

Im, K., Jo, H.J., Mangin, J.F., Evans, A.C., Kim, S.I., Lee, J.M., 2010. Spatial distribution of deep sulcal landmarks and hemispherical asymmetry on the cortical surface. Cerebral cortex 20, 602-11. URL: http://www. ncbi.nlm.nih.gov/pubmed/19561060, doi:10.1093/cercor/bhp127.

Im, K., Lee, J.M., Lee, J., Shin, Y.W., Kim, I.Y., Kwon, J.S., Kim, S.I., 2006. Gender difference analysis of cortical thickness in healthy young 
adults with surface-based methods. NeuroImage 31, 31-38. URL: http:// linkinghub.elsevier.com/retrieve/pii/S1053811905024900, doi:10. 1016/j.neuroimage.2005.11.042.

Im, K., Pienaar, R., Lee, J.M., Seong, J.K., Choi, Y.Y., Lee, K.H., Grant, P.E., 2011. Quantitative comparison and analysis of sulcal patterns using sulcal graph matching: a twin study. NeuroImage 57, 1077-86. URL: http://www.sciencedirect.com/science/article/ pii/S1053811911004794, doi:10.1016/j.neuroimage.2011.04.062.

Im, K., Pienaar, R., Paldino, M.J., Gaab, N., Galaburda, A.M., Grant, P.E., 2012. Quantification and discrimination of abnormal sulcal patterns in polymicrogyria. Cerebral cortex 23, 3007-15. URL: http://www.ncbi. nlm.nih.gov/pubmed/22989584.

Im, K., Raschle, N.M., Smith, S.A., Ellen Grant, P., Gaab, N., 2015. Atypical sulcal pattern in children with developmental dyslexia and at-risk kindergarteners. Cerebral Cortex URL: http://www. cercor.oxfordjournals . org/cgi/doi/10.1093/cercor/bhu305, doi:10.1093/cercor/bhu305.

Koenderink, J.J., 1984. The structure of images. Biological Cybernetics 50, 363-370. URL: http://dx.doi.org/10.1007/BF00336961, doi:10.1007/ BF00336961.

Kohavi, R., 1995. A study of cross-validation and bootstrap for accuracy estimation and model selection, Morgan Kaufmann. pp. 1137-1143.

Kriegeskorte, N., Goebel, R., Bandettini, P., 2006. Information-based functional brain mapping. Proceedings of the National Academy of Sciences of the United States of America 103, 3863-3868. URL: http: //www.pnas.org/content/103/10/3863. short.

Lindeberg, T., 1994. Scale-Space Theory in Computer Vision. Kluwer Academic Publishers, Norwell, MA, USA.

Lohmann, G., von Cramon, D.Y., Colchester, A.C.F., 2008. Deep sulcal landmarks provide an organizing framework for human cortical folding. Cerebral Cortex 18, 1415-1420. URL: http://cercor.oxfordjournals. org/content/18/6/1415. abstract, doi:10.1093/cercor/bhm174. 
Lv, B., Li, J., He, H., Li, M., Zhao, M., Ai, L., Yan, F., Xian, J., Wang, Z., 2010. Gender consistency and difference in healthy adults revealed by cortical thickness. NeuroImage 53, 373-382. URL: http:// linkinghub.elsevier.com/retrieve/pii/S1053811910007275, doi:10. 1016/j.neuroimage.2010.05.020.

Mangin, J.F., Rivière, D., Cachia, A., Duchesnay, E., Cointepas, Y., Papadopoulos-Orfanos, D., Collins, D.L., Evans, A.C., Régis, J., 2004. Object-based morphometry of the cerebral cortex. Medical Imaging, IEEE Transactions on 23, 968-982. URL: http://ieeexplore.ieee.org/xpls/ abs_all.jsp?arnumber=1318723.

Meng, Y., Li, G., Lin, W., Gilmore, J.H., Shen, D., 2014. Spatial distribution and longitudinal development of deep cortical sulcal landmarks in infants. NeuroImage 100, 206-218. URL: http:// linkinghub.elsevier.com/retrieve/pii/S1053811914004819, doi:10. 1016/j.neuroimage.2014.06.004.

Nichols, T.E., Holmes, A.P., 2002. Nonparametric permutation tests for functional neuroimaging: a primer with examples. Human brain mapping 15, 1-25. URL: http://onlinelibrary.wiley.com/doi/10.1002/hbm. $1058 /$ full.

Niederreiter, H., Sloan, I.H., 1994. Integration of nonperiodic functions of two variables by fibonacci lattice rules. Journal of Computational and Applied Mathematics 51, 57 - 70. URL: http://www.sciencedirect.com/ science/article/pii/037704279200004S, doi:http://dx.doi.org/10. 1016/0377-0427(92)00004-S.

Operto, G., Rivière, D., Fertil, B., Bulot, R., Mangin, J.F., Coulon, O., 2012. Structural analysis of fMRI data: A surface-based framework for multi-subject studies. Medical Image Analysis 16, 976-990. URL: http:// linkinghub.elsevier.com/retrieve/pii/S1361841512000357, doi:10. 1016/j.media.2012.02.007.

Poline, J.B., Mazoyer, B.M., 1993. Analysis of individual positron emission tomography activation maps by detection of high signal-to-noise-ratio pixel clusters. J Cereb Blood Flow Metab 13, 425-437. URL: http://dx.doi . org/10.1038/jcbfm.1993.57. 
Régis, J., Mangin, J.F., Ochiai, T., Frouin, V., Rivière, D., Cachia, A., Tamura, M., Samson, Y., 2005. Sulcal root generic model: a hypothesis to overcome the variability of the human cortex folding patterns. Neurologia medico-chirurgica 45,1-17. URL: http://www.ncbi.nlm.nih.gov/ pubmed/15699615.

Ruigrok, A.N., Salimi-Khorshidi, G., Lai, M.C., Baron-Cohen, S., Lombardo, M.V., Tait, R.J., Suckling, J., 2014. A meta-analysis of sex differences in human brain structure. Neuroscience \& Biobehavioral Reviews 39, 34-50. URL: http://linkinghub.elsevier.com/retrieve/ pii/S0149763413003011, doi:10.1016/j.neubiorev.2013.12.004.

Scholkopf, B., Smola, A.J., 2001. Learning with Kernels: Support Vector Machines, Regularization, Optimization, and Beyond. MIT Press, Cambridge, MA, USA.

Stelzer, J., Chen, Y., Turner, R., 2013. Statistical inference and multiple testing correction in classification-based multi-voxel pattern analysis (MVPA): Random permutations and cluster size control. NeuroImage 65, 69-82. URL: http://linkinghub.elsevier.com/retrieve/pii/ S1053811912009810, doi:10.1016/j.neuroimage.2012.09.063.

Sun, Z.Y., Klöppel, S., Rivière, D., Perrot, M., Frackowiak, R., Siebner, H., Mangin, J.F., 2012a. The effect of handedness on the shape of the central sulcus. NeuroImage 60, 332-339. URL: http:// linkinghub.elsevier.com/retrieve/pii/S1053811911014522, doi:10. 1016/j.neuroimage.2011.12.050.

Sun, Z.Y., Klöppel, S., Rivière, D., Perrot, M., Frackowiak, R.S.J., Siebner, H., Mangin, J.F., 2012b. The effect of handedness on the shape of the central sulcus. NeuroImage 60, 332-9. URL: http://www.ncbi.nlm.nih. gov/pubmed/22227053, doi:10.1016/j.neuroimage.2011.12.050.

Takerkart, S., Auzias, G., Brun, L., Coulon, O., 2015. Mapping cortical shape differences using a searchlight approach based on classification of sulcal pit graphs. Proceedings of IEEE ISBI Conference URL: https: //hal.archives-ouvertes.fr/hal-01112776/.

Takerkart, S., Auzias, G., Thirion, B., Ralaivola, L., 2014. Graph-based inter-subject pattern analysis of fMRI data. PloS ONE 9, e104586. doi:10. 1371/journal.pone.0104586. 
Toga, A.W., Thompson, P.M., 2003. Mapping brain asymmetry. Nat Rev Neurosci 4, 37-48. URL: http://dx.doi.org/10.1038/nrn1009, doi:10. $1038 / \mathrm{nrn} 1009$.

Van Essen, D.C., Drury, H.A., Dickson, J., Harwell, J., Hanlon, D., Anderson, C.H., 2001. An integrated software suite for surface-based analyses of cerebral cortex. Journal of the American Medical Informatics Association : JAMIA 8, 443-459. URL: http://www.ncbi.nlm.nih.gov/pmc/ articles/PMC131042/.

Van Essen, D.C., Glasser, M.F., Dierker, D.L., Harwell, J., Coalson, T., 2012. Parcellations and hemispheric asymmetries of human cerebral cortex analyzed on surface-based atlases. Cerebral Cortex 22, 2241-2262. URL: http://www.cercor.oxfordjournals.org/cgi/doi/ 10.1093/cercor/bhr291, doi:10.1093/cercor/bhr291.

Wu, G., Kim, M., Sanroma, G., Wang, Q., Munsell, B.C., Shen, D., 2015. Hierarchical multi-atlas label fusion with multi-scale feature representation and label-specific patch partition. NeuroImage 106, 34-46. URL: http:// linkinghub.elsevier.com/retrieve/pii/S1053811914009422, doi:10. $1016 / j$.neuroimage.2014.11.025.

Zhang, H., Nichols, T., Johnson, T., 2009. Cluster mass inference via random field theory. NeuroImage 44, 51-61. URL: http:// linkinghub.elsevier.com/retrieve/pii/S1053811908009117, doi:10. $1016 / j$.neuroimage.2008.08.017. 